\title{
Infinitesimal cohomology and the Chern character to negative cyclic homology
}

\author{
G. Cortiñas · C. Haesemeyer · C. A. Weibel
}

Received: 13 March 2007 / Revised: 24 July 2008 / Published online: 10 February 2009

(C) The Author(s) 2009. This article is published with open access at Springerlink.com

\begin{abstract}
There is a Chern character from $K$-theory to negative cyclic homology. We show that it preserves the decomposition coming from Adams operations, at least in characteristic zero.
\end{abstract}

\section{Introduction}

Shortly after the discovery of cyclic homology, Loday and others raised the question as to whether the Chern character $c h: K_{n}(A) \rightarrow H N_{n}(A)$ is compatible with the Adams operations $\psi^{k}[19,0.4 .1]$. Indeed, the respective Adams operations are defined in very different ways: for $K$-theory in [36,44,45], for cyclic homology in [17] and [39] and for negative cyclic homology $H N_{*}$ in [40] and [54].

G. Cortiñas' research was partially supported by grants ANPCyT PICT 2006-00836, UBACyT-X051, and MEC MTM 2007-64704. C. Haesemeyer's research was supported by NSF grant DMS-0813100.

C. A. Weibel's research was supported by NSA grant MSPF-04G-184, and by the Oswald Veblen Fund.

G. Cortiñas $(\varangle)$

Department of Matemática, FCEyN-UBA, Ciudad Universitaria Pab 1,

1428 Buenos Aires, Argentina

e-mail: gcorti@dm.uba.ar

C. Haesemeyer

Department of Mathematics, University of California Los Angeles, Box 951555, Los Angeles, CA 90095-1555, USA

e-mail: chh@math.ucla.edu

C. A. Weibel

Department of Mathematics, Rutgers University, New Brunswick, NJ 08901, USA

e-mail: weibel@math.rutgers.edu 
The following theorem, which we also prove for schemes of finite type over a field in Theorem 7.1 below, answers this in the affirmative for commutative rings $A$ containing $\mathbb{Q}$.

Theorem 0.1 For any commutative $\mathbb{Q}$-algebra $A$, the Chern character ch $: K_{n}(A) \rightarrow$ $H N_{n}(A)$ satisfies $\psi^{k} \operatorname{ch}(x)=k \operatorname{ch}\left(\psi^{k} x\right)$. That is, ch sends $K_{n}^{(i)}(A)$ to $H N_{n}^{(i)}(A)$.

Because $K^{(i)}(A)$ and $H N^{(i-1)}(A)$ are the eigenspaces for $\psi^{k}=k^{i}[40,4.5]$, the two formulations in this theorem are equivalent. The shift in indexing for $H N$ arises from the desire to have $\lambda^{k}=(-1)^{k-1} k^{i}$ on $H N^{(i)}(A)$ [40, 4.5.4].

Example 0.2 To see the difference in eigenvalues, consider the ring $R=F\left[t, t^{-1}\right]$. It is well known that the element $t \in K_{1}(R)$ satisfies $\psi^{k}(t)=k \cdot t$ for all $k$, while its character in $H N_{1}(R)$ satisfies $\psi^{k} \operatorname{ch}(t)=k^{2} \operatorname{ch}(t)$ [40, 8.4.7]. The image of $\operatorname{ch}(t)$ in $H H_{1}(R)=\Omega_{R}^{1}$ is $d t / t$, and again $\psi^{k}(d t / t)=k^{2}(d t / t)$.

Several special cases of this theorem have been addressed in the literature. The case of nilpotent ideals was asserted in the proof of $[17,7.5 .5]$ and established by Cathelineau in [6] (see our Appendix A). The case $K_{0}(A) \rightarrow H N_{0}(A)$, as well as the case $n<0$, was settled in [53], since $H N_{n}(A) \rightarrow H P_{n}(A)$ is an isomorphism for $n \leq 0$ and the composition $K_{n}(A) \rightarrow H N_{n}(A) \rightarrow H P_{n}(A)$ was shown to have this property in loc. cit. The Dennis trace map, which is the composition $K_{n}(A) \rightarrow H N_{n}(A) \rightarrow H H_{n}(A)$, was shown to be compatible with the Adams operations by Kantorowitz in [34]. Given the results of [12], Gillet and Soulé proved a very similar result in [20, 3.2.2 and 6.1], using the universal total Chern class.

Our method is to use infinitesimal cohomology to reduce the problem to the nilpotent case considered by Cathelineau. To that end, the goal of the first three sections is to interpret the infinitesimal cohomology $H^{*}\left(X_{\text {inf }}, H N\right)$ in terms of $H^{*}\left(X_{\text {inf }}, \mathcal{O}\right)$. In Sect. 1, we recall some basic facts about infinitesimal cohomology, introduced by Grothendieck in [27]. Section 2 reviews some elementary facts about pro-homological algebra which we need in Sect. 3 to prove a pro-version of the Hochschild-KostantRosenberg Theorem. The interpretation of $H^{n}\left(X_{\mathrm{inf}}, H N\right)$, and the fact that it vanishes for $n<0$, occurs in Example 3.8.

Section 4 introduces sheaf hypercohomology spectra for the infinitesimal topology, as a generalization of Grothendieck's construction. These ideas are applied to the $K$-theory spectrum in Sect. 5 , where the space $\mathbb{H}\left(X_{\text {inf }}, K\right)$ is compared to the fiber $K^{\text {inf }}$ of the Chern character. Here we establish the technical fact that $H N_{n}(X) \rightarrow$ $\pi_{n-1} \mathbb{H}\left(X_{\mathrm{inf}}, H N I\right)$ is an isomorphism for $n \leq-2$, and is injective for $n=-1$, where the presheaf $H N I$ on $X_{\text {inf }}$ is defined in Definition 5.1 so that $\mathbb{H}\left(X_{\text {inf }}, H N I\right)$ is the fiber of $\mathbb{H}\left(X_{\text {inf }}, H N\right) \rightarrow H N(X)$.

Section 6 extends Cathelineau's result to schemes, and to infinitesimal hypercohomology. Finally, our main theorem is proven in Sect. 7 as a special case of Theorem 7.1, which in turn follows from the technical fact mentioned above.

In Appendix A, we provide a technical correction to the preprint of [42], and to its use in the proof of Cathelineau's theorem in [6]. Although this correction is well known to the experts, it has not appeared in print before. In Appendix B, we give a simplicial presheaf version (and a spectrum version) of the same theorem. It is this 
version of Cathelineau's construction that we need in order to prove our main theorem. Appendix B depends upon some technical results about the model structure of simplicial presheaves (of sets); these results are proven in Appendix C.

Notation We shall write Sch $F$ for the category of schemes essentially of finite type over a field $F$. Objects of Sch $/ F$ shall be called $F$-schemes. If $k \subset F$ is a subfield, we write $\Omega_{/ k}^{p}$ for the sheaf of $p$-differential forms; we will write $\Omega^{p}$ when $k$ is clear from the context. If $H$ is a functor on $\mathrm{Sch} / F$ and $X=\operatorname{Spec}(A)$, we shall sometimes write $H(A)$ instead of $H(\operatorname{Spec} A)$; for example, $H^{*}\left(A, \Omega^{p}\right)$ is used for $H^{*}\left(\operatorname{Spec} A, \Omega^{p}\right)$.

We use cohomological indexing for all chain complexes in this paper; for a complex $C, C[p]^{q}=C^{p+q}$. For example, the Hochschild, cyclic, periodic and negative cyclic homology of schemes over a field $k$ (such as $F$-schemes over a field $F \supseteq k$ ) can be defined using the Zariski hypercohomology of certain presheaves of complexes; see [55] and $[12,2.7]$ for precise definitions. We shall write these presheaves as $H H(/ k)$, $H C(/ k), H P(/ k)$ and $H N(/ k)$, respectively, omitting $k$ from the notation if it is clear from the context.

If $\mathcal{E}$ is a presheaf of spectra on $\operatorname{Sch} / F$ (or just on $X$ ), we write $U \mapsto \mathbb{H}_{\text {zar }}(U, \mathcal{E})$ for Thomason's sheaf hypercohomology spectrum [49, 1.33]. Jardine showed in [30, 3.3] that $\mathbb{H}_{\text {zar }}(-, \mathcal{E})$ is the fibrant replacement for $\mathcal{E}$ in the model structure of [31, 1.5]; see [50, D.5]. We say that $\mathcal{E}$ satisfies Zariski descent on Sch $/ F$ (or on $X$ ) if the natural maps $\mathcal{E}(U) \rightarrow \mathbb{H}_{\text {zar }}(U, \mathcal{E})$ are homotopy equivalences for all $U$ in $\mathrm{Sch} / F$ (resp., $U \subset X$ ).

It is well known $[54,10.9 .19]$ that there is an Eilenberg-Mac Lane functor $C \mapsto|C|$ from chain complexes of abelian groups to spectra, and from presheaves of chain complexes of abelian groups to presheaves of spectra. This functor sends quasiisomorphisms of complexes to weak homotopy equivalences of spectra, and satisfies $\pi_{n}(|C|)=H^{-n}(C)$; the loop space is $\Omega|C| \simeq|C[-1]|$. For example, applying $\pi_{n}$ to the Chern character $K \rightarrow|H N|$ yields maps $K_{n}(A) \rightarrow H^{-n} H N(A)=H N_{n}(A)$.

\section{Infinitesimal cohomology of sheaves}

Recall from [27] that a closed immersion of schemes $U \hookrightarrow T$ is called a thickening of $U$ if its ideal of definition is nilpotent; by abuse of notation, we write $T$ to mean $U \hookrightarrow T$. For $X \in \mathrm{Sch} / F$, the infinitesimal site $X_{\mathrm{inf}}$ consists of the category $\inf (X)$ and its coverings, which we now define. Objects of $\inf (X)$ are thickenings $U \hookrightarrow T$, where $U$ is an open subscheme of $X$; morphisms from $U \hookrightarrow T$ to $U^{\prime} \hookrightarrow T^{\prime}$ are morphisms $T \rightarrow T^{\prime}$ in Sch $/ F$ under inclusions $U \subseteq U^{\prime}$. A covering of $T$ is a family of morphisms $\left\{T_{i} \rightarrow T\right\}$ such that the $T_{i}$ form a Zariski open covering of $T$.

A sheaf $\mathcal{E}$ on $X_{\text {inf }}$ is the same thing as a compatible collection of Zariski sheaves $\left\{\mathcal{E}_{T} \in \operatorname{Sh}(T): T \in \inf (X)\right\}$ (see [3, Sect. 5], [27, 4.1]). It follows that $X_{\text {inf }}$ has enough points, namely the Zariski points on the thickenings $T$.

Note that by definition, the forgetful functor

$$
u: \inf (X) \rightarrow \operatorname{Sch} / F, \quad(U \hookrightarrow T) \mapsto T,
$$


is a morphism of sites $(\mathrm{Sch} / F)_{\mathrm{zar}} \rightarrow X_{\mathrm{inf}}$. Thus if $\mathcal{E}$ is a Zariski sheaf on $\mathrm{Sch} / F$, its restriction to $\inf (X)$ defines a sheaf $u^{*} \mathcal{E}$ on $X_{\text {inf }}$; by abuse, we will write $\mathcal{E}$ for $u^{*} \mathcal{E}$. The usual global sections functor takes a sheaf $\mathcal{E}$ on $X_{\text {inf }}$ to:

$$
H^{0}\left(X_{\mathrm{inf}}, \mathcal{E}\right)=\Gamma_{\mathrm{inf}}(\mathcal{E})=\lim _{T \in \inf (X)} H^{0}\left(T, \mathcal{E}_{T}\right)
$$

and the infinitesimal cohomology of a sheaf of abelian groups is defined as:

$$
H^{*}\left(X_{\mathrm{inf}}, \mathcal{E}\right)=H^{*} \mathbb{H}\left(X_{\mathrm{inf}}, \mathcal{E}\right), \quad \text { where } \mathbb{H}\left(X_{\mathrm{inf}}, \mathcal{E}\right)=\mathbb{R} \Gamma_{\mathrm{inf}}(\mathcal{E}) .
$$

Example 1.2 For affine $X=\operatorname{Spec}(A)$, we can compute infinitesimal cohomology using the method outlined by Grothendieck in [27, p. 338]. Suppose for simplicity that $\mathcal{E}$ is a quasi-coherent sheaf on $\mathrm{Sch} / F$, and that the natural map $j^{*} \mathcal{E}_{T^{\prime}} \rightarrow \mathcal{E}_{T}$ is an isomorphism for every closed embedding $j: T \hookrightarrow T^{\prime} \operatorname{in} \inf (X)$; in this case $\mathcal{E}$ is called a crystal; see [3, 2.12].

Let $A=S / I_{1}$ be a presentation of $A$ as a quotient of a smooth $F$-algebra $S$, and write $I_{v}$ for the kernel of $S^{\otimes v} \rightarrow A$. Then each $S_{\bullet}^{\otimes v}=\left\{S^{\otimes v} / I_{v}^{m}\right\}_{m}$ is a tower of algebras, and $[v] \mapsto\left\{S_{\bullet}^{\otimes v+1}\right\}$ is a cosimplicial tower of algebras. Following [27], set $Y_{m}^{v}=\operatorname{Spec}\left(S^{\otimes v} / I_{v}^{m}\right)$. Further, define $\mathcal{E}\left(Y_{\bullet}^{v}\right)=\lim _{m} \mathcal{E}\left(Y_{m}^{v}\right)$. Regarding the cosimplicial group $\mathcal{E}\left(Y_{\bullet}^{*+1}\right)$ as a cochain complex, we have:

$$
H^{*}\left(X_{\mathrm{inf}}, \mathcal{E}\right)=H^{*} \mathcal{E}\left(Y_{\bullet}^{*+1}\right)
$$

Here is a modern interpretation of Grothendieck's argument in [27, 5.2]. The functor $\Gamma_{\mathrm{inf}}$ factors as the forgetful functor $\Gamma_{\mathrm{zar}}$ from sheaves to presheaves, followed by the inverse limit functor. The forgetful functor preserves injectives as it is right adjoint to sheafification. Following [4, XI.6] and [49, 5.32], we write $\operatorname{holim}_{T}$ for $\mathbb{R} \lim _{T \in \inf (X)}$ and $\mathbb{H}_{\text {zar }}(T, \mathcal{E})$ for $\mathbb{R} \Gamma_{\text {zar }}(T, \mathcal{E})$. It follows from [54, 10.8.3] that we have an isomorphism in the derived category:

$$
\mathbb{H}\left(X_{\mathrm{inf}}, \mathcal{E}\right) \simeq \operatorname{holim}_{T} \mathbb{H}_{\mathrm{zar}}(T, \mathcal{E})
$$

Lemma 1.4 Suppose that $X=\operatorname{Spec}(A)$ and $A=S / I$ for a smooth algebra $S$. Then the simplicial cotower $[\nu] \mapsto\left\{Y_{\bullet}^{\nu+1}\right\}$ of Example 1.2 is right cofinal in $\inf (X)$.

Right cofinality means that for each object $T$ of $\inf (X)$, the category $T / i$ is contractible, where $i$ denotes the inclusion of the simplicial cotower into inf $(X)$.

Proof By $[9,5.1]$, the simplicial cotower is right cofinal in the subcategory $X / \inf (X)$ of thickenings of $X$. We assert that the proof of $[9,5.1]$ goes through mutatis mutandis. Given a thickening $U \hookrightarrow T$, set $B=\mathcal{O}(T), C=\mathcal{O}(U)$ and pick $r$ so that $\operatorname{ker}(B \rightarrow C)^{r}=0$. Consider the cosimplicial object $h_{r}: \Delta \rightarrow \inf (X)^{o p}$ defined by the $Y_{r}^{v+1}$.

As in op. cit., the inclusion $T / h_{r} \subset T / i$ is a homotopy equivalence, $\left(T / h_{r}\right)^{o p}$ is the homotopy colimit of the discrete simplicial set $\operatorname{Hom}_{\inf (X)}\left(T, Y_{r}^{*+1}\right)$, and this simplicial set is contractible. Since $T / h_{r}$ is contractible, so is $T / i$. 
Let $[v] \mapsto C_{v}$ be a cosimplicial abelian group or, more generally, a cosimplicial complex of abelian groups. Recall from $[49,5.32]$ that the Bousfield-Kan total complex holim ${ }_{v \in \Delta} C_{v}^{*}=\operatorname{Tot}_{v} C_{v}^{*}$ is just a specific Cartan-Eilenberg resolution of the associated total cochain complex $C^{*}$.

Proposition 1.5 Suppose that $X=\operatorname{Spec}(A)$ and that $\mathcal{E}$ is a cochain complex of quasi-coherent sheaves on $\mathrm{Sch} / F$.

1. $\operatorname{holim}_{T} \mathcal{E}(T) \simeq \operatorname{Tot}_{v}\left(\mathbb{R} \lim _{m}\right) \mathcal{E}\left(Y_{m}^{\nu+1}\right)$.

2. Assume that $j^{*} \mathcal{E}_{T^{\prime}} \rightarrow \mathcal{E}_{T}$ is onto for every closed embedding $j: T \hookrightarrow T^{\prime}$ in $\inf (X)$. Writing $\mathcal{E}\left(Y_{\bullet}^{v}\right)$ for $\lim _{m} \mathcal{E}\left(Y_{m}^{v}\right)$ as in Example 1.2 , we have: $\mathbb{H}\left(X_{\mathrm{inf}}, \mathcal{E}\right) \simeq$ $\operatorname{Tot}_{v} \mathcal{E}\left(Y_{\bullet}^{v+1}\right)$.

Proof By Lemma 1.4 and the cofinality theorem [4, XI.9.2], holim $_{T}$ is equivalent to $\operatorname{holim}_{\mathbb{N} \times \Delta}=\operatorname{holim}_{\Delta} \operatorname{holim}_{\mathbb{N}}=\operatorname{Tot}\left(\mathbb{R} \lim _{m}\right)$. Part (1) follows because, by hypothesis, $\mathcal{E}(T)=\mathbb{H}_{\text {zar }}(T, \mathcal{E})$ for all affine $T$. Under the assumptions of $(2), \mathbb{R}_{\lim _{m}} \mathcal{E}\left(Y_{m}^{v}\right)$ can be replaced by $\lim _{m} \mathcal{E}\left(Y_{m}^{v}\right)=\mathcal{E}\left(Y_{\bullet}^{v}\right)$.

Remark 1.6 Let $k \subset F$ be a subfield. The sheaf $\Omega^{p}=\Omega_{/ k}^{p}$ of $p$-differential forms is not a crystal for $p \neq 0$ in the sense of Example 1.2. However, it satisfies the hypotheses of Proposition 1.5(2), allowing us to compute $\mathbb{H}\left(X_{\mathrm{inf}}, \Omega^{p}\right)$ using Tot $_{v}$.

Let $\mathcal{E}$ be a cochain complex of sheaves of abelian groups on $\inf (X)$.

Lemma 1.7 The presheaf $V \mapsto \mathbb{H}\left(V_{\mathrm{inf}}, \mathcal{E}\right)$ satisfies Zariski descent on $X$.

Proof For every Zariski open $j: V \subset X$, the inclusion $\inf (V) \subset \inf (X)$ has a right adjoint $\rho: \inf (X) \rightarrow \inf (V)$, sending thickenings of $U$ to thickenings of $U \cap V$. For simplicity, we shall write $T \cap V$ for the thickening of $U \cap V$ corresponding to $T$, so that $\rho(U \hookrightarrow T)$ is $U \cap V \hookrightarrow T \cap V$. By Lemma 1.4, the Bousfield-Kan cofinality theorem [4, XI.9.2] applies to say that the map of Cartan-Eilenberg resolutions

$$
\underset{T \in \inf (X)}{\operatorname{holim}} \mathbb{H}_{\text {zar }}(T \cap V, \mathcal{E}) \rightarrow \underset{\inf (V)}{\operatorname{holim}} \mathbb{H}_{\text {zar }}(T, \mathcal{E})
$$

is a quasi-isomorphism. Now if $\left\{V_{1}, V_{2}\right\}$ is a Zariski cover of an open $V$, we have a distinguished triangle for every $T \operatorname{in} \inf (V)$ :

$$
\mathbb{H}_{\text {zar }}(T, \mathcal{E}) \rightarrow \mathbb{H}_{\text {zar }}\left(T \cap V_{1}, \mathcal{E}\right) \times \mathbb{H}_{\text {zar }}\left(T \cap V_{2}, \mathcal{E}\right) \rightarrow \mathbb{H}_{\text {zar }}\left(T \cap V_{1} \cap V_{2}, \mathcal{E}\right)
$$

Taking the homotopy limit over $T$, we obtain the triangle

$$
\mathbb{H}\left(V_{\mathrm{inf}}, \mathcal{E}\right) \rightarrow \mathbb{H}\left(V_{1 \mathrm{inf}}, \mathcal{E}\right) \times \mathbb{H}\left(V_{2 \mathrm{inf}}, \mathcal{E}\right) \rightarrow \mathbb{H}\left(\left(V_{1} \cap V_{2}\right)_{\mathrm{inf}}, \mathcal{E}\right)
$$

which implies the assertion that $\mathbb{H}\left(-{ }_{\text {inf }}, \mathcal{E}\right)$ satisfies Zariski descent on $X$.

Remark 1.8 Formula (1.7a) says that for any Zariski open $j: V \subset X$ and any complex of presheaves of abelian groups $\mathcal{E}$ on $\inf (V)$, the direct image $j_{*} \mathcal{E}(T)=\mathcal{E}(T \cap V)$ satisfies $\mathbb{H}\left(V_{\mathrm{inf}}, \mathcal{E}\right) \cong \mathbb{H}\left(X_{\mathrm{inf}}, j_{*} \mathcal{E}\right)$. 
Here is an extension of Grothendieck's theorem [27, 4.1]:

Theorem 1.9 The brutal truncations $\Omega^{*} \rightarrow \Omega \leq i \rightarrow \Omega^{0}=\mathcal{O}$ induce homotopy equivalences

$$
\mathbb{H}\left(X_{\mathrm{inf}}, \Omega^{*}\right) \simeq \mathbb{H}\left(X_{\mathrm{inf}}, \Omega^{\leq i}\right) \simeq \mathbb{H}\left(X_{\mathrm{inf}}, \mathcal{O}\right)
$$

Proof From the hypercohomology spectral sequence (see [54, 5.7.9])

$$
E_{1}^{p, q}=H^{q}\left(X_{\mathrm{inf}}, \Omega^{p}\right) \Rightarrow H^{p+q}\left(X_{\mathrm{inf}}, \Omega^{*}\right)
$$

we see that it suffices to prove that $\mathbb{H}\left(X_{\mathrm{inf}}, \Omega^{p}\right)=0$ for $p>0$. By induction on the size of a separated cover, using Lemma 1.7, we are reduced to the case in which $X$ is separated. A similar induction reduces us to the case in which $X$ is affine.

The affine case was established in [11, 7.9] using the method of Example 1.2 above. As argued in loc. cit., it suffices to show that $\left\{\Omega_{S^{\otimes \bullet} / I_{\bullet}}^{p}\right\}_{\nu}$ is acyclic, where $S$ is a symmetric algebra. This complex is the inverse limit of the tower

$$
\left\{\Omega_{S^{\otimes \bullet}}^{p} / I_{\bullet}^{m} \Omega_{S^{\otimes \bullet}}^{p}\right\}_{v}
$$

When $I=0$, a more or less explicit chain contraction is given in loc. cit., and is defined by differential operators.

Since a contraction of this kind must be continuous for the $I_{\bullet}$-adic topology, the result follows.

Associated to the de Rham mixed complex $\left(\Omega_{R}, 0, d\right)$ is the Connes double complex $H C \Omega_{R}$ for cyclic homology; see [40, 2.5.10], [54, 9.8.8]. We can also form double complexes $H P \Omega_{R}$ and $H N \Omega_{R}$ for periodic and negative cyclic homology. The following is immediate from Theorem 1.9 and the hypercohomology spectral sequence for the row filtration on these double complexes.

Corollary 1.10 For any $X$ in $\mathrm{Sch} / F$,

$$
\mathbb{H}\left(X_{\mathrm{inf}}, H C \Omega\right) \simeq \prod_{i \geq 0} \mathbb{H}\left(X_{\mathrm{inf}}, \mathcal{O}\right)[2 i]
$$

Similarly, for the periodic and negative cyclic variants we have

$$
\begin{aligned}
\mathbb{H}\left(X_{\mathrm{inf}}, H P \Omega\right) & \simeq \prod_{i \in \mathbb{Z}} \mathbb{H}\left(X_{\mathrm{inf}}, \mathcal{O}\right)[2 i] \\
\text { and } \mathbb{H}\left(X_{\mathrm{inf}}, H N \Omega\right) & \simeq \prod_{i \leq 0} \mathbb{H}\left(X_{\mathrm{inf}}, \mathcal{O}\right)[2 i]
\end{aligned}
$$




\section{Pro-homological algebra}

In order to prove the main result (Theorem 3.2) in the next section, we need some elementary results on the homological algebra of pro-objects. No great originality is claimed for the results in this section.

We recall from [1, A.4.5] that if $\mathcal{A}$ is an abelian category then the pro-category pro $-\mathcal{A}$ is also abelian, and so is the full subcategory of $\operatorname{pro}-\mathcal{A}$ consisting of towers $\left\{A_{m}\right\}=\left\{A_{m}\right\}_{m}$ (indexed by the natural numbers $m \geq 0$ ).

A tower $\left\{A_{m}\right\}$ is isomorphic to 0 in $\operatorname{pro}-\mathcal{A}$ if and only if it satisfies the trivial Mittag-Leffler condition that for every $m$ there exists a $j>m$ such that $A_{j} \rightarrow A_{m}$ is zero. A strict morphism of towers $\left\{f_{m}\right\}:\left\{A_{m}\right\} \rightarrow\left\{B_{m}\right\}$ is an isomorphism in pro-A if and only if the kernel $\left\{\operatorname{ker}\left(f_{m}\right)\right\}$ and cokernel $\left\{\operatorname{coker}\left(f_{m}\right)\right\}$ are isomorphic to 0 in $\operatorname{pro}-\mathcal{A}$.

If $\left\{i_{m}\right\}$ is a strictly increasing sequence of natural numbers then $\left\{A_{i_{m}}\right\} \rightarrow\left\{A_{m}\right\}$ is an isomorphism in pro-A $\mathcal{A}$, and every morphism $\left\{A_{i}\right\} \rightarrow\left\{B_{m}\right\}$ in pro-A is represented by a strict morphism of towers $\left\{A_{i_{m}}\right\} \rightarrow\left\{B_{m}\right\}$.

If $R$ is a ring, $M$ an $R$-module, and $J \subset R$ an ideal, we write $M / J^{\infty} M$ for the pro- $R$-module $\left\{M / J^{m} M\right\}_{m}$.

Lemma 2.1 Let $R$ be a noetherian ring and $J$ an ideal. Then $M \mapsto M / J^{\infty} M$ is an exact functor from the category of finitely generated $R$-modules to pro-R-modules.

Proof Suppose that $0 \rightarrow L \rightarrow M \rightarrow N \rightarrow 0$ is an exact sequence of finitely generated $R$-modules. Then $M / J^{\infty} M \rightarrow N / J^{\infty} N$ is onto with kernel $\left\{L / J^{m} M \cap L\right\}$. By the Artin-Rees lemma, $\left\{J^{m} L\right\} \rightarrow\left\{J^{m} M \cap L\right\}$ is a pro-isomorphism. It follows that $\left\{L / J^{m} L\right\} \rightarrow\left\{L / J^{m} M \cap L\right\}$ is also a pro-isomorphism.

Example 2.2 If $I$ is an ideal in a ring $S$, then the pro- $R$-modules $\Omega_{S}^{p} / I^{\infty} \Omega_{S}^{p}$ and $\left\{\Omega_{S / I^{m}}^{p}\right\}$ are isomorphic for all $p$, with the isomorphism coming from the Fundamental Exact Sequence

$$
I / I^{2} \rightarrow \Omega_{S} / I \Omega_{S} \rightarrow \Omega_{S / I} \rightarrow 0
$$

In particular, $\lim _{\leftarrow} \Omega_{S}^{p} / I^{m} \Omega_{S}^{p}=\lim _{\leftarrow} \Omega_{S / I^{m}}^{p}$

Lemma 2.3 Let $R$ be a ring, $J \subset R$ an ideal and $f: M \rightarrow N$ a surjective homomorphism of $R$-modules. Let $P:=\left\{P_{m}\right\}$ be a pro-R-module such that each $P_{m}$ is a projective $R / J^{m}$-module. Given any morphism $g: P \rightarrow N / J^{\infty} N$ of pro-R-modules, there exists a pro-homomorphism $h: P \rightarrow M / J^{\infty} M$ making the following diagram commute.

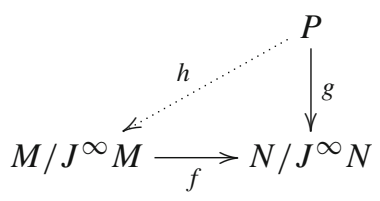


Proof Choose a representative $\left\{g_{m}: P_{i_{m}} \rightarrow N / J^{m} N\right\}$ of $g$. Then each $Q_{m}=$ $P_{i_{m}} / J^{m} P_{i_{m}}$ is a projective $R / J^{m}$-module, and there is a pro-module isomorphism $\left\{Q_{m}\right\} \cong\left\{P_{m}\right\}$.

Replacing $\left\{P_{m}\right\}$ by $\left\{Q_{m}\right\}$ if necessary, we may assume that $\left\{g_{m}: P_{m} \rightarrow N / J^{m} N\right\}$ is a strict map.

We will construct a strict lift $\left\{h_{m}\right\}$ by induction on $m$. The case $m=1$ is clear because $P_{1}$ is a projective $R / J$-module. Inductively, we have a lift $h_{m}: P_{m} \rightarrow$ $M / J^{m} M$ of $g_{m}$ and hence a map $P_{m+1} \rightarrow M / J^{m} M \times_{N / J^{m} N} N / J^{m+1} N$. Since $M / J^{m+1} \rightarrow M / J^{m} M \times_{N / J^{m}} N N / J^{m+1} N$ is onto and $P_{m+1}$ is projective, we get the desired lift $P_{m+1} \rightarrow M / J^{m+1}$ compatible with $h_{m}$ and $g_{m+1}$.

Corollary 2.4 Let $R$ be a noetherian ring, $M$ a finitely generated $R$-module, and $\epsilon: L_{*} \rightarrow M$ a resolution by finitely generated $R$-modules. Let $J \subset R$ be an ideal. Then $L_{*} / J^{\infty} L_{*} \rightarrow M / J^{\infty} M$ is a resolution in the category of pro- $R$-modules.

If in addition $P_{*}=\left\{P_{*, m}\right\}_{m}$ is a chain complex of pro- $R$-modules, such that each $P_{n, m}$ is a projective $R / J^{m}$-module, and $P_{*} \rightarrow M / J^{\infty} M$ is a chain map of promodules, then there is a chain map of pro-modules $P_{*} \rightarrow L_{*} / J^{\infty} L$, unique up to pro-chain homotopy, making the following diagram commute.

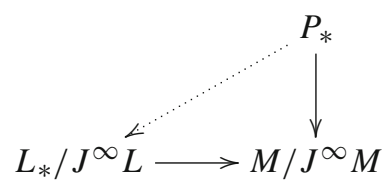

Proof The first assertion is just Lemma 2.1. Given this, the proof of the usual Comparison Theorem [54, 2.2.6] goes through using Lemma 2.3.

Lemma 2.5 Let $R$ be a ring and let $r_{1}, \ldots, r_{n} \in R$ be such that $R=\left(r_{1}, \ldots, r_{n}\right) R$. Then for every pro-R-module $M=\left\{M_{m}\right\}, M$ is pro-isomorphic to 0 if and only if each $M\left[1 / r_{i}\right]$ is.

Proof Straightforward, using the trivial Mittag-Leffler condition.

If $\left\{P_{*}=P_{*, m}\right\}$ is a pro-chain complex, meaning that $P_{*, m}$ is a chain complex for each $m$ and the $P_{*, m+1} \rightarrow P_{*, m}$ are chain maps, then we may regard $P$ as a chain complex of pro-objects. Conversely, any bounded below chain complex of pro-objects is pro-isomorphic to a pro-chain complex via the re-indexing trick.

Lemma 2.6 Let $R$ be a ring, $J \subset R$ an ideal, $P_{*}$ and $Q_{*}$ two bounded below procomplexes of $R$-modules, and $f: P_{*} \rightarrow Q_{*}$ a homomorphism of complexes of pro-R-modules. Assume that at each level $m$ and each degree $n$, both $P_{n, m}$ and $Q_{n, m}$ are $R / J^{m}$-modules, that $P_{n, m}$ is projective, and that each complex $Q_{*, m}$ is acyclic.

Then for every additive functor $F$ from $R$-modules to an abelian category $\mathcal{A}$, and each $n$, the induced map $H_{n}\left(F\left(P_{*}\right)\right) \rightarrow H_{n}\left(F\left(Q_{*}\right)\right)$ is zero in pro-A.

Proof We claim that by re-indexing $P_{*}$ we may assume that $f$ is a strict chain map of towers. In this case, because each $P_{*, m}$ is a complex of projectives and each $Q_{*, m}$ is 
acyclic, each $f_{m}$ is chain-homotopic to zero by the usual Comparison Theorem [54, 2.2.6], and the result follows.

To see the claim, note that we can choose a function $h: \mathbb{N}^{2} \rightarrow \mathbb{N}$, strictly increasing with respect to each variable separately, together with a representative $f_{n, m}$ : $P_{n, h(n, m)} \rightarrow Q_{n, m}$, so that the following diagram commutes for all $n$ and $m:$

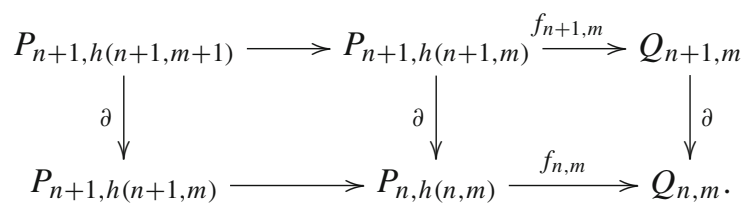

(We have abused notation by omitting all notation for transition maps $P_{*, j} \rightarrow P_{*, i}$.) Set $P_{n, m}^{\prime}=P_{n, h(n, m)} / J^{m} P_{n, h(n, m)}$; this is a projective $R / J^{m}$-module. Because $P_{*}$ is a pro-complex, $P_{*}^{\prime}$ is a tower of chain complexes, pro-isomorphic to $P_{*}$, and the induced map $P_{*}^{\prime} \rightarrow Q_{*}$ is a strict map of towers of complexes.

\section{Pro-Hochschild-Kostant-Rosenberg theorem}

Let $F$ be a field, $S$ an algebra over $F$, and $I \subset S$ an ideal. Write $\Omega$ and $H H$ for $\Omega_{/ F}^{*}$ and Hochschild homology taken over $F$, respectively. Then the shuffle product [54, 9.4.4] induces a map of graded pro- $S$-modules:

$$
\left\{\Omega_{S / I^{m}}^{p}\right\}_{m} \rightarrow\left\{H H_{p}\left(S / I^{m}\right)\right\}_{m}
$$

The purpose of this section is to prove the following theorem for fields $F$ of arbitrary characteristic, and its analogue for subfields $k \subseteq F$ in characteristic 0 .

Theorem 3.2 If $S$ is essentially of finite type and smooth over $F$ then (3.1) is a proisomorphism for every ideal $I \subset S$.

The proof of Theorem 3.2 which we shall give is an adaptation to the pro-setting of the proof of the Hochschild-Kostant-Rosenberg theorem [28] [54, 9.4.7]. In the proof, all vector spaces, tensor products, algebras, and differential forms will be taken over $F$.

Proof Let $\mathcal{J}=\operatorname{ker}\left(S^{e} \rightarrow S\right)$, where $S^{e}=S \otimes S$. Because $\mathcal{J} / \mathcal{J}^{2}=\Omega_{S}^{1}$ is projective, $\mathcal{J}$ is locally a complete intersection. Thus we can find elements $\alpha_{1}, \ldots, \alpha_{n} \in S^{e}$ such that $\operatorname{Spec}(S)=V(\mathcal{J}) \subset \bigcup_{i} \operatorname{Spec} S^{e}\left[1 / \alpha_{i}\right]$ and such that for each $i$, the ideal $\mathcal{J}\left[1 / \alpha_{i}\right] \subset S^{e}\left[1 / \alpha_{i}\right]$ is a complete intersection. Let $s_{i}$ be the image of $\alpha_{i}$ under the map $S^{e} \rightarrow S$; we have $S^{e}\left[1 / \alpha_{i}\right] / \mathcal{J}\left[1 / \alpha_{i}\right]=S\left[1 / s_{i}\right]$, and Spec $S=\bigcup_{i}$ Spec $S\left[1 / s_{i}\right]$. Upon replacing $\alpha_{i}$ by $\left(s_{i} \otimes s_{i}\right) \alpha_{i}$ if necessary, we may assume that $S^{e}\left[1 / \alpha_{i}\right]$ is a localization of $S\left[1 / s_{i}\right]^{e}$. Lemma 2.5 applied to the pro- $S$-modules given by the kernel and cokernel of the map (3.1) shows that it suffices to check that the latter becomes an isomorphism after inverting each $s_{i}$. Fixing $i$, replacing $S$ by $S\left[1 / s_{i}\right]$, and setting 
$\alpha=\alpha_{i}$, we may therefore assume that $\mathcal{J}_{\alpha}=\mathcal{J}[1 / \alpha]$ is a complete intersection in $S_{\alpha}^{e}=S^{e}[1 / \alpha]$, and that $S=S[1 / \alpha]$. Put

$$
J=(S \otimes I+I \otimes S)[1 / \alpha] \text { and } J^{(m)}=\left(S \otimes I^{m}+I^{m} \otimes S\right)[1 / \alpha] .
$$

Note that $J^{(m)} \subset J^{m}$ and $J^{2 m} \subset J^{(m)}$, so $J^{(\bullet)}$ and $J^{\bullet}$ are equivalent filtrations and the pro-algebra map $\left\{\left(S / I^{m}\right)^{e}=S^{e} / J^{(m)}\right\} \rightarrow\left\{S^{e} / J^{\infty}\right\}$ is an isomorphism.

Consider the bar resolution $C_{*}^{\text {bar }}(S) \stackrel{\sim}{\longrightarrow} S$ of $S$ as a $S^{e}$-module; it has

$$
C_{n}^{b a r}(S)=S^{e} \otimes S^{\otimes n}=S^{\otimes n+2} \quad(n \geq 0) .
$$

Because $S_{\alpha}^{e}$ is a localization of $S^{e}, S_{\alpha}^{e} \otimes S^{e} C_{*}^{b a r}(S)$ is an $S_{\alpha}^{e}$-projective resolution of $S$. Since $S_{\alpha}^{e} / J^{(m)}=\left(S / I^{m}\right)_{\alpha}^{e}$,

$$
Q_{*, m}=S_{\alpha}^{e} \otimes_{S^{e}} C_{*}^{b a r}\left(S / I^{m}\right)=\left(S / I^{m}\right)_{\alpha}^{e} \otimes_{S^{e}} C_{*}^{b a r}\left(S / I^{m}\right)
$$

is a projective $\left(S / I^{m}\right)_{\alpha}^{e}$-module resolution of $S / I^{m}$. Similarly, its quotient

$$
P_{*, m}=S_{\alpha}^{e} / J^{m} \otimes_{S^{e}} C_{*}^{b a r}\left(S / I^{m}\right)
$$

is a complex of projective $S_{\alpha}^{e} / J^{m}$-modules. Setting $S_{\alpha}^{e} / J^{(\infty)}=\left\{S_{\alpha}^{e} / J^{(m)}\right\}$ we have an isomorphism of pro-complexes

$$
Q_{*}=S_{\alpha}^{e} / J^{(\infty)} \otimes_{S^{e}} C_{*}^{b a r}\left(S / I^{\infty} S\right) \stackrel{\sim}{\longrightarrow} S_{\alpha}^{e} / J^{\infty} \otimes_{S^{e}} C_{*}^{\text {bar }}\left(S / I^{\infty} S\right)=P_{*} .
$$

Choose $x_{1}, \ldots, x_{d} \in \mathcal{J}$ whose images in $J_{\alpha}$ form a regular sequence of generators, and write $L_{*}$ for the Koszul complex $K\left(S_{\alpha}^{e} ; x_{1}, \ldots, x_{d}\right)$. Then $L_{*}$ is an $S_{\alpha}^{e}$-projective resolution of $S$, of the form $L_{*}=S_{\alpha}^{e} \otimes \wedge^{*} V$, where $V$ is a $d$-dimensional $F$-vector space with basis $\left\{v_{1}, \ldots, v_{d}\right\}$, and $L_{1} \rightarrow L_{0}$ sends $v_{i}$ to $x_{i}$.

By the Comparison Theorem, there is a chain equivalence $L_{*} \stackrel{\epsilon}{\longrightarrow} S_{\alpha}^{e} \otimes C_{*}^{b a r}(S)$ of projective resolutions of $S$, unique up to chain homotopy. We can in fact choose $\epsilon$ to induce the map (3.1). To do so, write $x_{i}=\sum_{j} r_{i j}\left(s_{i j} \otimes 1-1 \otimes s_{i j}\right)$, with $r_{i j}, s_{i j} \in S$, and set $\epsilon\left(v_{i}\right)=\sum_{j} r_{i j} \otimes s_{i j} \otimes 1$. This defines a map $V \rightarrow S_{\alpha}^{e} \otimes S^{e} C_{1}^{b a r}(S)$, and we extend it to an $S_{\alpha}^{e}$-linear homomorphism of chain complexes using the shuffle product of $C_{*}^{\text {bar }}(S)$.

Composing $\epsilon$ with $S_{\alpha}^{e} \otimes_{S^{e}} C_{*}^{b a r}(S) \rightarrow P_{*, m}$ induces maps $L_{*} / J^{m} L_{*} \rightarrow P_{*, m}$ and hence a strict map of pro-complexes

$$
\epsilon: L / J^{\infty} L \rightarrow P_{*}
$$

which covers the identity of $S / I^{\infty} S$. Since $S / I^{m}=S \otimes S_{\alpha}^{e} S_{\alpha}^{e} / J^{m}$, tensoring (3.2b) over $S_{\alpha}^{e}$ with $S$ and using Example 2.2, we obtain a chain map of pro-complexes

$$
\begin{aligned}
& \left\{\Omega_{S / I^{m}}\right\}_{m} \cong \Omega_{S} / I^{\infty} \Omega_{S}=S / I^{\infty} \otimes \wedge^{*} V \\
& \quad=S \otimes S^{e} L / J^{\infty} L \stackrel{\epsilon_{S}}{\longrightarrow} S \otimes S^{e} P_{*} \cong\left\{S / I^{m} \otimes_{S^{e}} C_{*}^{b a r}\left(S / I^{m}\right)\right\}_{m}
\end{aligned}
$$


where the boundary operator of the first two complexes is the zero map. We observe that the homology of the right side computes $H H_{*}\left(S / I^{\infty}\right)$, and that (3.2c) induces the same map in homology as the map (3.1) (obtained from the shuffle product).

We shall prove that (3.2c) is a quasi-isomorphism. By Corollary 2.4, we have a map

$$
\mu: P_{*}=S_{\alpha}^{e} / J^{\infty} \otimes_{S^{e}} C_{*}^{b a r}\left(S / I^{\infty}\right) \rightarrow L / J^{\infty} L
$$

which again covers the identity of $S / I^{\infty} S$. Note moreover that

$$
L_{0} / J^{\infty} L_{0}=S_{\alpha}^{e} / J^{\infty}=S_{\alpha}^{e} / J^{\infty} \otimes S^{e} C_{0}^{b a r}\left(S / I^{\infty} S\right)
$$

so we can take $\mu$ to be the identity in degree zero. By the uniqueness in Corollary 2.4, we obtain an $S_{\alpha}^{e}$-linear homotopy $\mu \epsilon \rightarrow 1_{L / J^{\infty} L}$. Applying $S \otimes_{S_{\alpha}^{e}}$ and taking homology in (3.2c), we obtain that (3.1) is a monomorphism in pro-homology.

To prove that (3.1) is also surjective we note that, by (3.2a), we may augment the pro-complex $Q_{*}$ to a pro-acyclic complex by adding $S / I^{\infty} S$ in degree -1 . Now consider the map $\left\{P_{*, 2 m}\right\}_{m} \rightarrow\left\{Q_{*, m}\right\}$ which is zero in degrees $-1,0$ and agrees with $\epsilon \mu-1$ in higher degrees. Applying Lemma 2.6 to this map (relative to the ideal $J^{2}$ ), we see that the composition with (3.2a) is zero on homology. Since this map is $\epsilon \mu-1$, $\epsilon \mu$ is the identity map on homology, as required.

Note that the map $\mu$ constructed in the proof depends critically upon the ArtinRees Lemma for $\mathcal{J}$, even in degree 2 . The following example illustrates Theorem 3.2 in a simple case. Set $\Lambda_{m}=F[x] /\left(x^{m+1}\right)$ and $\Lambda_{m}^{R}=R[x] /\left(x^{m+1}\right)$. It is well known that $H H_{n}\left(\Lambda_{m}\right) \cong \Lambda_{m-1}$ for all $n>0$. By the Künneth formula, $H H_{*}\left(\Lambda_{m}^{R}\right)=$ $H H_{*}(R) \otimes_{F} H H_{*}\left(\Lambda_{m}\right)$.

Lemma 3.3 Let $F$ be a field of characteristic zero. For any $F$-algebra $R$ and for all $M>2 m$, the image of $H H_{n}\left(\Lambda_{M}^{R}\right) \rightarrow H H_{n}\left(\Lambda_{m}^{R}\right)$ is

$$
\left(H H_{n}(R) \otimes \Lambda_{m}\right) \oplus\left(H H_{m-1}(R) \otimes \Omega_{\Lambda_{m} / F}^{1}\right)
$$

In particular, if $R$ is smooth over $F$, then the image is

$$
\Omega_{\Lambda_{m}^{R} / F}^{n}=\left(\Omega_{R / F}^{n} \otimes_{F} \Lambda_{m}\right) \oplus\left(\Omega_{R}^{n-1} \otimes_{F} \Omega_{\Lambda_{m} / F}^{1}\right) .
$$

Proof Explicit $\Lambda_{m}$-module generators were given in $[18,1.10]$ for $H H_{*}\left(\Lambda_{m}\right)$ in terms of $u=[x]$ and of the sum $t_{m}$ of all terms $x^{a}\left[x^{b} \mid x\right]$ with $a+b=m$, considered as elements of the bar complex: $H H_{2 i}\left(\Lambda_{m}\right)$ is generated by $x t_{m}^{i}$, and $H H_{2 i+1}\left(\Lambda_{m}\right)$ is generated by $u t_{m}^{i}$. If $M>2 m$, every term in $t_{M}$ contains an $x^{m+1}$ factor and so vanishes in the bar complex of $\Lambda_{m}$. It follows that the map $H H_{n}\left(\Lambda_{M}\right) \rightarrow H H_{n}\left(\Lambda_{m}\right)$ is zero for all $n>1$. By the Künneth formula, the map from $H H_{n}\left(R \otimes_{F} \Lambda_{M}\right)$ to $H H_{n}\left(R \otimes_{F} \Lambda_{m}\right)$ vanishes on all summands except for $H H_{n}(R) \otimes_{F} \Lambda_{M}$ and $H H_{n-1}(R) \otimes_{F} \Omega_{\Lambda_{M} / F}^{1}$, where it is the natural surjection.

We can use the following spectral sequence to replace $F$ by any subfield $k$ in the statement of Theorem 3.2. 
Lemma 3.4 [35, 4.3a] Let $k \subseteq F$ be fields of characteristic zero. For each $p \geq 1$ there is a bounded second quadrant homological spectral sequence $(0 \leq i<p, j \geq 0)$ :

$$
{ }_{p} E_{-i, i+j}^{1}=\Omega_{F / k}^{i} \otimes_{F} H H_{p-i+j}^{(p-i)}(R / F) \Rightarrow H H_{p+j}^{(p)}(R / k)
$$

Proposition 3.5 Let $k \subset F$ be fields of characteristic zero, $S$ an algebra over $F$, essentially of finite type and smooth, and $I \subset S$ an ideal. Write $\Omega$ for $\Omega_{/ k}$. For each $p \geq 0$, the shuffle product induces an isomorphism of pro-S-modules

$$
\left\{\Omega_{S / I^{m}}^{p}\right\}_{m} \rightarrow\left\{H H_{p}\left(\left(S / I^{n}\right) / k\right)\right\}_{m}
$$

Proof Applying the natural, uniformly bounded spectral sequence of Lemma 3.4 levelwise, we obtain a bounded spectral sequence in the category of pro- $S$-modules, which converges to the pro-Hochschild homology over $k$ of $S / I^{\infty}$. It follows from Theorem 3.2 that the latter spectral sequence degenerates, being zero for $j \neq 0$, proving the result.

For any $\mathbb{Q}$-algebra $R$, let $(C(R), b, B)$ be the usual cyclic mixed complex of $R$; we have a canonical map $e:(C(R), b, B) \rightarrow\left(\Omega_{R}, 0, d\right)[54,9.8 .12]$.

Proposition 3.7 The map of mixed complexes $e:(C, b, B) \rightarrow(\Omega, 0, d)$ induces an equivalence of fibration sequences for every $X$ in $\mathrm{Sch} / F$ :

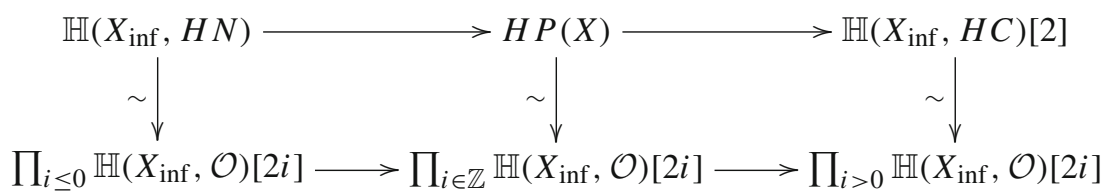

Proof The top row is $\mathbb{H}\left(X_{\mathrm{inf}},-\right)$ applied to the triangle $H N \rightarrow H P \rightarrow H C$ [2], using Example 4.6 below. By Corollary 1.10, the bottom row is $\mathbb{H}\left(X_{\text {inf }},-\right)$ applied to the triangle $H N \Omega \rightarrow H P \Omega \rightarrow H C \Omega$ [2]. The vertical maps are the maps $e$, so the diagram commutes. It remains to show that $e$ induces equivalences. For this, we may assume that $X$ is affine by repeated applications of Lemma 1.7.

Consider the induced morphism $e: \mathbb{H}\left(X_{\text {inf }}, \mathcal{E}\right) \rightarrow \mathbb{H}\left(X_{\text {inf }}, \mathcal{E} \Omega\right)$, where $\mathcal{E}$ is $H C$, $H P$ or $H N$. By Remark 1.6 and Proposition 1.5(2), $e$ is Tot $_{v}$ applied to the map $\lim _{m} \mathcal{E}\left(Y_{m}^{v+1}\right) \rightarrow \lim _{m}(\mathcal{E} \Omega)\left(Y_{m}^{v+1}\right)$, which is a weak equivalence by (3.6).

Example 3.8 Taking cohomology in Proposition 3.7, we see that $H^{n}\left(X_{\text {inf }}, H N\right)$ is: 0 for $n<0 ; H^{0}\left(X_{\mathrm{inf}}, \mathcal{O}\right)$ for $n=0$; and for $n>0$ it is the (finite) product

$$
H^{n}\left(X_{\mathrm{inf}}, H N\right)=\prod_{0 \leq j \leq n / 2} H^{n-2 j}\left(X_{\mathrm{inf}}, \mathcal{O}\right) .
$$




\section{Infinitesimal hypercohomology spectra}

In this section, we rework the homological material of Sect. 1 in the context of presheaves of spectra. We need this generality in order to form the infinitesimal hypercohomology spectrum for $K$-theory, introduced in Sect. 5. The main result of this section (Theorem 4.9) is that our construction is the categorical hypercohomology spectrum, i.e., global sections of the fibrant replacement functor.

Definition 4.1 Let $\mathcal{E}$ be a presheaf of spectra on $X_{\text {inf }}$. We define $\mathbb{H}\left(X_{\text {inf }}, \mathcal{E}\right)$ to be the homotopy limit

$$
\mathbb{H}\left(X_{\text {inf }}, \mathcal{E}\right)=\operatorname{holim}_{T \in \inf (X)} \mathbb{H}_{\text {zar }}(T, \mathcal{E})
$$

By construction [4, XI.3.4], there is a canonical map $\mathbb{H}\left(X_{\text {inf }}, \mathcal{E}\right) \rightarrow \mathbb{H}_{\text {zar }}(X, \mathcal{E})$. Since this spectrum definition is parallel to the homological construction in (1.3), we see that $\mathbb{H}\left(X_{\text {inf }}, \mathcal{E}\right)$ agrees with the homological definition (1.1) when $\mathcal{E}$ is an EilenbergMac Lane spectra associated to a complex of sheaves.

Example 4.2 For all $X$ in $\mathrm{Sch} / F$, we see from Proposition 3.7 and 3.8 that

$$
\mathbb{H}\left(X_{\text {inf }}, H N\right) \cong \prod_{j \geq 0} \Omega^{2 j} \mathbb{H}\left(X_{\text {inf }}, \mathcal{O}\right)
$$

In particular, if $n>0$ then $\pi_{n} \mathbb{H}\left(X_{\mathrm{inf}}, H N\right)=0$, and $\pi_{0} \mathbb{H}\left(X_{\text {inf }}, H N\right)=\mathbb{H}^{0}\left(X_{\text {inf }}, \mathcal{O}\right)$.

Because holim and $\mathbb{H}_{\text {zar }}(X,-)$ preserve fibration sequences, we have:

Lemma 4.3 $\mathbb{H}\left(X_{\mathrm{inf}},-\right)$ preserves fibration sequences.

We say that $\mathcal{E}$ is nilinvariant on $X_{\text {zar }}$ if it takes thickenings $U \hookrightarrow T$ to weak equivalences for every open $U$ in $X$.

Example 4.4 For example, $\mathbb{H}\left(-{ }_{\text {inf }}, \mathcal{E}\right)$ is nilinvariant on $X_{\text {zar }}$ because for any thickening $X \hookrightarrow X^{\prime}$ the map $\inf \left(X^{\prime}\right) \rightarrow \inf (X)$ has a left adjoint (the pushout of $U \hookrightarrow T$ along the unique $\left.U \subset U^{\prime}\right)$, so that holim $\operatorname{inf(X^{\prime })}$ and $\operatorname{holim}_{\inf (X)}$ are weak equivalent.

Lemma 4.5 If $\mathcal{E}$ is nilinvariant on $X_{\mathrm{zar}}$, and satisfies Zariski descent on $X$, then

$$
\mathbb{H}\left(X_{\text {inf }}, \mathcal{E}\right) \cong \mathcal{E}(X)
$$

Proof By nilinvariance, $\mathcal{E}(T) \simeq \mathcal{E}(U)$ for any infinitesimal thickening $U \hookrightarrow T$ in $\inf (X)$. Therefore $\mathbb{H}\left(X_{\text {inf }}, \mathcal{E}\right) \cong \operatorname{holim}_{U \in X_{\text {zar }}} \mathcal{E}(U)$. By Zariski descent, this homotopy limit is $\mathbb{H}_{\text {zar }}(X, \mathcal{E}) \simeq \mathcal{E}(X)$.

Example 4.6 Periodic cyclic homology $H P$ is nilinvariant (see Goodwillie [24] [54, 9.9.9]), and satisfies Zariski descent (see [12, 2.9]). Thus $\mathbb{H}\left(X_{\mathrm{inf}}, H P\right) \cong H P(X)$.

Here is the spectrum analogue of Example 1.2 and Proposition 1.5, which says that when $X=\operatorname{Spec}(A)$ is affine, the groups $\mathbb{H}^{*}\left(X_{\mathrm{inf}}, \mathcal{E}\right)$ may be computed using the thickenings $Y_{m}^{v}=\operatorname{Spec}\left(S^{\otimes v} / I_{v}^{m}\right)$ constructed in Example 1.2. 
Lemma 4.7 Let $\mathcal{E}$ be a presheaf of spectra on $\mathrm{Sch} / \mathrm{F}$ satisfying Zariski descent. Then for each $F$-algebra A of finite type, presented as in Example 1.2:

$$
\mathbb{H}\left((\operatorname{Spec} A)_{\mathrm{inf}}, \mathcal{E}\right) \simeq \underset{v}{\operatorname{Tot}} \mathcal{E}\left(Y_{\bullet}^{\nu+1}\right) .
$$

Proof Set $X=\operatorname{Spec}(A)$. By Definition 4.1, $\mathbb{H}\left(X_{\text {inf }}, \mathcal{E}\right) \simeq \operatorname{holim}_{T} \mathcal{E}(T)$, and each $\mathcal{E}(T)$ is a fibrant spectrum; our goal is to interpret the homotopy limit via Tot. Let $A=S / I_{1}$ be a presentation of $A$ as a quotient of a smooth $F$-algebra. As in Example 1.2, we form the cosimplicial tower of algebras $S_{\bullet}^{\otimes \nu+1}$ and the simplicial cotower $Y_{\bullet}^{v}$ in the category inf $(X)$.

By Lemma 1.4, Bousfield-Kan cofinality [4, XI.9.2] and [4, XI.4.1-4.3],

$$
\underset{T}{\operatorname{holim}} \mathcal{E}(T) \simeq \underset{\Delta}{\operatorname{holim}} \operatorname{holim}_{\mathbb{N} o p} \mathcal{E}\left(Y_{m}^{v+1}\right) \simeq \underset{\Delta}{\operatorname{holim}} \mathcal{E}\left(Y_{\bullet}^{v+1}\right)
$$

As in 1.5, but using [4, XI.4.4], this is $\operatorname{Tot}_{v}$ of $\mathcal{E}\left(Y_{\bullet}^{\otimes v+1}\right)$.

Now we compare the $\mathbb{H}\left(X_{\mathrm{inf}},-\right)$ construction of Definition 4.1 with the categorical hypercohomology construction of [49] and [30]. As one might expect, it turns out that infinitesimal hypercohomology can be computed as global sections of a fibrant replacement in an appropriate model structure.

Recall from [31] that there is a "local injective" model structure on presheaves of spectra on any site, and in particular on $\inf (X)$. A map $\mathcal{E} \rightarrow \mathcal{E}^{\prime}$ is an (infinitesimal) local weak equivalence if it induces an isomorphism on sheaves of homotopy groups; it is a cofibration if each $\mathcal{E}(T) \rightarrow \mathcal{E}^{\prime}(T)$ is a cofibration of spectra in the sense of [5]; fibrations are defined by the right lifting property.

Proposition 4.8 For any local weak equivalence $\mathcal{E} \rightarrow \mathcal{E}^{\prime}$ of presheaves of spectra on $\inf (X)$, the map $\mathbb{H}\left(X_{\mathrm{inf}}, \mathcal{E}\right) \rightarrow \mathbb{H}\left(X_{\mathrm{inf}}, \mathcal{E}^{\prime}\right)$ is a weak equivalence of spectra.

Proof By the discussion in Sect. 1, the hypothesis means that the stalks of $\pi_{*} \mathcal{E}$ and $\pi_{*} \mathcal{E}^{\prime}$ are isomorphic at every Zariski point $t \in T$, for any $U \hookrightarrow T$ in $X_{\text {inf }}$. Fixing $T$, this shows that $\mathcal{E}_{T} \rightarrow \mathcal{E}_{T}^{\prime}$ is a Zariski local weak equivalence in $T$, and hence that $\mathbb{H}_{\mathrm{zar}}(T, \mathcal{E}) \rightarrow \mathbb{H}_{\mathrm{zar}}\left(T, \mathcal{E}^{\prime}\right)$ is a homotopy equivalence. By [4, XI.5.6], $\operatorname{holim}_{T}$ preserves homotopy equivalences, so $\mathbb{H}\left(X_{\mathrm{inf}}, \mathcal{E}\right) \simeq \mathbb{H}\left(X_{\mathrm{inf}}, \mathcal{E}^{\prime}\right)$.

Recall that a fibrant replacement of $\mathcal{E}$ is a cofibration $\mathcal{E} \rightarrow \mathcal{E}^{\prime}$ with $\mathcal{E}^{\prime}$ fibrant which is a weak equivalence. Because the $T$ form a covering sieve of the terminal sheaf $*$ on $X_{\text {inf }}$, the global sections spectrum of $\mathcal{E}^{\prime}$ is

$$
\operatorname{hom}_{*}\left(*, \mathcal{E}^{\prime}\right)=\operatorname{hom}_{*}\left(\underline{\lim }_{T} T, \mathcal{E}^{\prime}\right)=\lim _{T} \operatorname{hom}_{*}\left(T, \mathcal{E}^{\prime}\right)=\lim _{T} \mathcal{E}^{\prime}(T) .
$$

Here $\operatorname{hom}_{*}\left(T, \mathcal{E}^{\prime}\right)$ denotes the usual spectrum Hom from a presheaf of sets into a spectrum, and $T$ is regarded as a (representable) presheaf of sets.

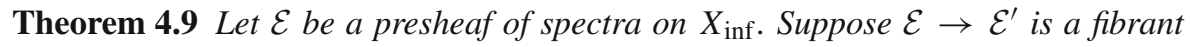
replacement. Then $\mathbb{H}\left(X_{\mathrm{inf}}, \mathcal{E}\right) \cong \operatorname{hom}_{*}\left(*, \mathcal{E}^{\prime}\right)$. 
Proof By Proposition $4.8, \mathbb{H}\left(X_{\mathrm{inf}}, \mathcal{E}\right) \simeq \mathbb{H}\left(X_{\text {inf }}, \mathcal{E}^{\prime}\right)$, so we may assume $\mathcal{E}=\mathcal{E}^{\prime}$

For any $U \subseteq T$ in $\inf (X)$, consider the functor $t: \operatorname{zar}(T) \rightarrow \inf (X)$ sending a Zariski open $T^{\prime} \subseteq T$ to the evident thickening of $U \times_{T} T^{\prime}$. It induces a morphism of topoi $t_{*}$, from sheaves on $X_{\text {inf }}$ to Zariski sheaves on $T$. Now $t_{*}$ preserves globally fibrant objects by [32, p.119], so we consider $\mathcal{E}_{T}^{\prime}=t_{*}\left(\mathcal{E}^{\prime}\right)$.

Since globally fibrant presheaves on $T$ satisfy Zariski descent [30], it follows that $\mathcal{E}^{\prime}(T)=\mathcal{E}_{T}^{\prime}(T) \simeq \mathbb{H}_{\mathrm{zar}}\left(T, \mathcal{E}_{T}^{\prime}\right)$ and hence that $\mathbb{H}\left(X_{\mathrm{inf}}, \mathcal{E}^{\prime}\right) \simeq \operatorname{holim}_{T} \mathcal{E}^{\prime}(T)$.

Consider the covering sieve $\{T\}$ of $*$, the terminal sheaf on $X_{\text {inf }}$. By [33, p.367 and Lemma 11], hocolim $T \rightarrow *$ is a local weak equivalence. Combining this with the fact that $\mathcal{E}^{\prime}$ is fibrant, the fact that $\mathcal{E}^{\prime}(T)=\operatorname{hom}_{*}\left(T, \mathcal{E}^{\prime}\right)$ for $T \in \inf (X)$, and adjointness [4, XII.4.1], we obtain homotopy equivalences:

$$
\begin{aligned}
& \operatorname{hom}_{*}\left(*, \mathcal{E}^{\prime}\right) \simeq \operatorname{hom}_{*}\left(\operatorname{hocolim}_{T} T, \mathcal{E}^{\prime}\right) \\
& \quad \cong \operatorname{holim}_{T} \operatorname{hom}_{*}\left(T, \mathcal{E}^{\prime}\right)=\mathbb{H}\left(X_{\mathrm{inf}}, \mathcal{E}^{\prime}\right) .
\end{aligned}
$$

Remark 4.10 Fibrant presheaves are not always nilinvariant. In particular, $\mathcal{E}^{\prime}(X)$ is not always equivalent to $\mathbb{H}\left(X_{\text {inf }}, \mathcal{E}^{\prime}\right) \cong \operatorname{hom}_{*}\left(*, \mathcal{E}^{\prime}\right)$.

\section{Infinitesimal $K$-theory}

In this section, we apply the infinitesimal hypercohomology construction to the algebraic $K$-theory spectrum.

Definition 5.1 For $U \subset T \operatorname{in} \inf (X)$, let $K I(T)$ and $H N I(T)$ denote the respective homotopy fibers of $K(T) \rightarrow K(U)$ and $H N(T) \rightarrow H N(U)$. The relative Chern character induces a natural map $K I(T) \rightarrow H N I(T)$. Goodwillie's theorem [26] states that $K I(T) \rightarrow H N I(T)$ is a homotopy equivalence. (It is well known that the $\pi_{n} K I(T)$ are uniquely divisible; see [52]).

Remark 5.2 Let $K(\mathcal{O}, I)$ and $H N(\mathcal{O}, I)$ denote the presheaves sending $U \subset T$ to the fibers of $K(\mathcal{O}(T)) \rightarrow K(\mathcal{O}(U))$ and $H N(\mathcal{O}(T)) \rightarrow H N(\mathcal{O}(U))$. Then $K(\mathcal{O}, I) \rightarrow$ $K I$ and $H N(\mathcal{O}, I) \rightarrow H N I$ are local weak equivalences on $\inf (X)$, because every thickening is locally affine. By Proposition $4.8, \mathbb{H}\left(X_{\text {inf }}, K(\mathcal{O}, I)\right) \simeq \mathbb{H}\left(X_{\text {inf }}, K I\right)$ and $\mathbb{H}\left(X_{\mathrm{inf}}, H N(\mathcal{O}, I)\right) \simeq \mathbb{H}\left(X_{\mathrm{inf}}, H N I\right)$.

Theorem 5.3 The map $\mathbb{H}\left(X_{\mathrm{inf}}, K I\right) \rightarrow \mathbb{H}\left(X_{\mathrm{inf}}, H N I\right)$ is a homotopy equivalence for all $X$ in $\mathrm{Sch} / F$, and there is a commutative diagram whose rows are fibrations:

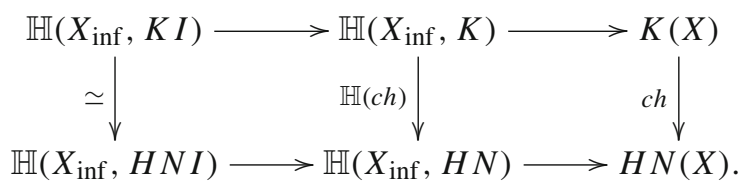

Proof Applying $\mathbb{H}\left(X_{\mathrm{inf}},-\right)$ to $K I(T) \stackrel{\simeq}{\longrightarrow} H N I(T)$ yields the first assertion. 
By Lemma 4.5, $\mathbb{H}\left(X_{\text {inf }}, T \mapsto K(U)\right) \cong K(X)$, and similarly for $T \mapsto H N(U)$. By Lemma 4.3, applying $\mathbb{H}\left(X_{\mathrm{inf}},-\right)$ to $K I(T) \rightarrow K(T) \rightarrow K(U)$ and its $H N$ analogue yields the desired equivalence of fibration sequences.

Theorem 5.4 If $n \geq 1$ then $H N_{n}(X) \cong \pi_{n-1} \mathbb{H}\left(X_{\text {inf }}, K I\right)$ for all $X$ in $\mathrm{Sch} / F$.

Proof The result is immediate from Theorem 5.3 when $n>1$, since $\pi_{n} \mathbb{H}\left(X_{\text {inf }}\right.$, $H N)=0$ for $n \geq 1$ by Example 4.2. For $n=1$ it suffices to show that $\pi_{0} \mathbb{H}\left(X_{\text {inf }}\right.$, $H N) \rightarrow H N_{0}(X)$ is an injection. In fact,

$$
\pi_{n} \mathbb{H}\left(X_{\mathrm{inf}}, H N\right) \rightarrow H N_{n}(X) \rightarrow H P_{n}(X)
$$

is a split injection for all $n$, by Proposition 3.7.

Remark 5.5 When $n \leq 0, \pi_{n-1} \mathbb{H}\left(X_{\text {inf }}, K I\right) \cong \prod_{i>0} \mathbb{H}^{2 i-n}\left(X_{\text {inf }}, \mathcal{O}\right)$ for affine $X$. This follows from Theorem 5.3, Proposition 3.7 and $H N_{n}(X)=H P_{n}(X) \cong$ $\prod_{i} \mathbb{H}^{2 i-n}\left(X_{\mathrm{inf}}, \mathcal{O}\right)$.

Remark 5.6 Although the setup is analogous to that of [9] and [10], those articles allow noncommutative thickenings, and [9] uses connective $K$-theory. These differences account for the restriction $n \geq 1$ in Theorem 5.4. Nevertheless, up to these slight differences in definitions, Theorem 5.4 recovers the second main result, Theorem 6.2(i), of [9]. That is, for $n \geq 1$ the Chern character $K_{n}(X) \rightarrow H N_{n}(X)$ may be indentified with the map $K_{n}(X) \rightarrow \pi_{n-1} \mathbb{H}\left(X_{\text {inf }}, K I\right)$ in Theorem 5.3. This fails for $n=0$, since the second map omits the "rank" component

$$
c h_{0}: K_{0}(X) \rightarrow H^{0}(X, \mathbb{Z}) \rightarrow H^{0}\left(X_{\text {inf }}, \mathcal{O}\right)
$$

of the Chern character $K_{0}(X) \rightarrow H N_{0}(X)$, as Remark 5.5 shows.

We conclude this section with a comparison (Theorem 5.9) between infinitesimal hypercohomology $\mathbb{H}\left(X_{\text {inf }}, K\right)$ and the "infinitesimal $K$-theory" used in our earlier papers [12] and [13] and based upon the construction in the eponymous paper [9].

Definition 5.7 Let $K^{\text {inf }}(X)$ denote the homotopy fiber of the Chern character $c h$ : $K(X) \rightarrow H N(X)$. Here $K(X)$ is non-connective $K$-theory, not the connected and rational version used in [9].

Lemma $5.8 \mathbb{H}\left(X_{\text {inf }}, K^{\mathrm{inf}}\right) \simeq K^{\mathrm{inf}}(X)$.

This lemma is immediate from Theorem 5.3 and Lemma 4.3. Alternatively, note that $K^{\text {inf }}$ satisfies Zariski descent, because both $K$ and $H N$ do. Moreover, $K^{\text {inf }}$ is nilinvariant, by Definition 5.1, so Lemma 5.8 also follows from Lemma 4.5.

Theorem 5.9 For any $X \in \mathrm{Sch} / F$, there is a homotopy fibration sequence:

$$
K^{\mathrm{inf}}(X) \rightarrow \mathbb{H}\left(X_{\mathrm{inf}}, K\right) \rightarrow \prod_{i \geq 0} \Omega^{2 i} \mathbb{H}\left(X_{\mathrm{inf}}, \mathcal{O}\right)
$$

In particular, for $n \geq 1, K_{n}^{\mathrm{inf}}(X) \cong \pi_{n} \mathbb{H}\left(X_{\mathrm{inf}}, K\right)$. 
Proof Applying $\mathbb{H}\left(X_{\mathrm{inf}},-\right)$ to the fibration of Definition 5.7, and using Lemmas 5.8 and 4.3 , we get a fibration sequence $K^{\mathrm{inf}}(X) \rightarrow \mathbb{H}\left(X_{\mathrm{inf}}, K\right) \rightarrow \mathbb{H}\left(X_{\mathrm{inf}}, H N\right)$. The sequence in 5.6 and the homotopy group calculation follow from Example 4.2.

Remark 5.10 One should compare Theorem 5.9 to [9, Theorem 6.2(ii)]. This is not straightforward, because our notation is not compatible with that in [9].

\section{Cathelineau's Theorem for Schemes}

In this section, we develop both a scheme-theoretic version (6.1) and an infinitesimal version (6.3) of Cathelineau's Theorem (see Theorems A.1 and B.8 in the Appendices). Although we do not need the scheme-theoretic version for our Main Theorem 0.1 , it is of independent interest. It also sets the stage for the infinitesimal version, which we will need for Theorem 7.1, and hence for our Main Theorem. Throughout this section, we work over a field of characteristic 0 .

Let $I$ be a nilpotent sheaf of ideals on a scheme, and consider the Zariski presheaf $K(-, I)$, sending $U$ to the homotopy fiber of $K(U) \rightarrow K(U / I)$, where $U / I$ is the scheme over $U$ with structure sheaf $\mathcal{O}_{U} / I$. By [50], $K$ and $K(-, I)$ satisfy Zariski descent. Since $K(\mathcal{O}, I) \rightarrow K(-, I)$ is a local weak equivalence for the Zariski topology, it follows that we have $K(U, I) \simeq \mathbb{H}_{\mathrm{zar}}(U, K(\mathcal{O}, I))$.

By B.10, both $\lambda^{k}$ and $\psi^{k}$ are spectrum maps from $K(\mathcal{O}, I)$ to itself. Applying Zariski descent, they induce spectrum maps from each $K(U, I)$ to itself.

We define $H C(-, I)$ and $H N(-, I)$ similarly, or as the Eilenberg-Mac Lane spectra associated to the appropriate chain complexes of sheaves. Since $\lambda^{k}$ and $\psi^{k}$ are associated to chain maps, compatible with the homotopy equivalences $B: H C(U, I)$ $[1] \rightarrow H N(U, I)$, they are spectrum maps compatible with $B$.

Theorem 6.1 If I is a nilpotent sheaf of ideals on a scheme, then the relative Chern character $K(-, I) \rightarrow H C(-, I)[1] \simeq H N(-, I)$ is compatible with the operations $\lambda^{k}$ in the sense that $\psi^{k}(\operatorname{ch} x)=k \cdot \operatorname{ch}\left(\psi^{k} x\right)$ for each $x \in K_{m}(U, I)$.

In addition, we have a homotopy commutative diagram of presheaves of spectra:

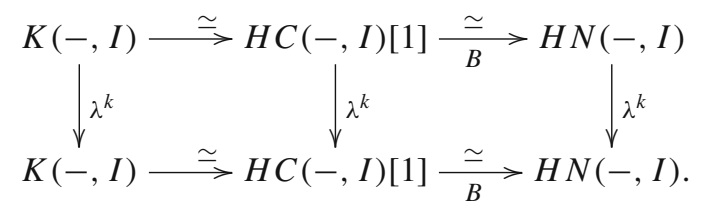

Proof By the spectrum version B.10 of Theorem B.8, we have a homotopy commutative diagram of presheaves of spectra:

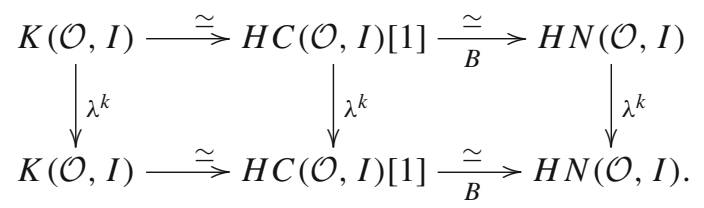


Applying Zariski hypercohomology (and homotopy groups), we get the result.

Let $K^{(i)}(-, I), H C^{(i-1)}(-, I)$ and $H N^{(i-1)}(-, I)$ denote the respective homotopy fibers of $\psi^{k}-k^{i}$ on $K(-, I), H C(-, I)$ and $H N(-, I)$. Copying the proof of Corollary B.12 proves the following.

Corollary 6.2 There is a homotopy commutative diagram for each $U$ :

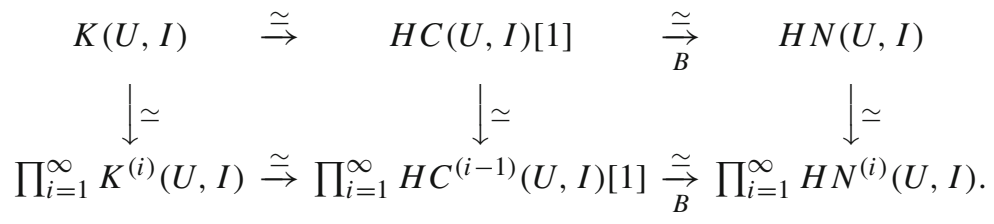

We now turn to the analogues of Theorem 6.1 and Corollary 6.2 for the infinitesimal topology on a fixed $X$ in $\mathrm{Sch} / F$. In order to distinguish the tautological ideal of a thickening $U \subset T$ from the Zariski case, we adopt the notation $K I$ and $H N I$ from Definition 5.1 rather than the notation $K(-, I)$ and $H N(-, I)$ used above.

The relative Chern character $c h: K I \rightarrow H N I$ induces an infinitesimal Chern character $\mathbb{H}\left(X_{\mathrm{inf}}, K I\right) \rightarrow \mathbb{H}\left(X_{\mathrm{inf}}, H N I\right)$, and we saw in Theorem 5.3 that it is a homotopy equivalence. Let $K I^{(i)}(T)$ and $H N I^{(i)}(T)$ denote the respective homotopy fibers of $\psi^{k}-k^{i}$ on $K I$ and $H N I$; as in Theorem B.11 these are defined using a fixed $k \geq 2$, but are independent of this choice up to homotopy equivalence.

Theorem 6.3 For any $X$ in $\mathrm{Sch} / F$, the infinitesimal Chern character is compatible with the operations $\lambda^{k}$. That is, we have a commutative diagram of spectra:

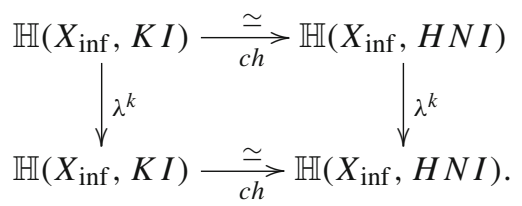

There is also a homotopy commutative diagram:

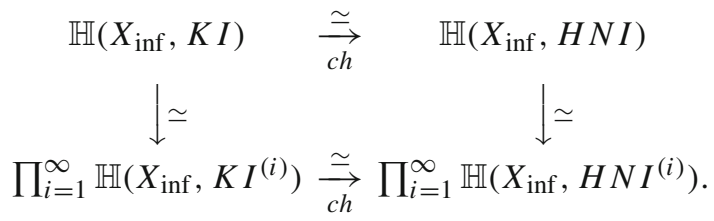

Proof The maps $K(\mathcal{O}, I) \rightarrow K I$ and $H N(\mathcal{O}, I) \rightarrow H N I$ are local weak equivalences on $X_{\text {inf }}$ by Remark5.2, and are compatible with the operations $\psi^{k}$. Hence they induce local weak equivalences $K^{(i)}(\mathcal{O}, I) \rightarrow K I^{(i)}$ and $H N^{(i)}(\mathcal{O}, I) \rightarrow H N I^{(i)}$. By Proposition 4.8, the diagrams in question are weak equivalent to the functorial infinitesimal hypercohomology construction 4.1 applied to the commutative diagrams in Theorem B.8, Corollary B.10 and Corollary B.12. 


\section{Hodge decomposition}

We now turn to the proof of the main theorem 0.1 stated in the introduction. We first prove the result for $n \geq 1$, and for all $X$ in $\operatorname{Sch} / F$, where $F$ is a field of characteristic 0 .

Theorem 7.1 For all $X$ in $\mathrm{Sch} / F$ and all $n \geq 1$, the map ch $: K_{n}(X) \rightarrow H N_{n}(X)$ satisfies $\psi^{k}($ ch $)=k \cdot \operatorname{ch}\left(\psi^{k}\right)$, i.e., ch sends $K_{n}^{(i)}(X)$ to $H N_{n}^{(i)}(X)$.

Proof The left half of the following diagram commutes by Theorem 5.3, and the right half of this diagram commutes by Theorem 6.3. The lower left map is injective when $n \geq 1$ because in that case $\pi_{n} \mathbb{H}\left(X_{\text {inf }}, H N\right)=0$ by Example 4.2.

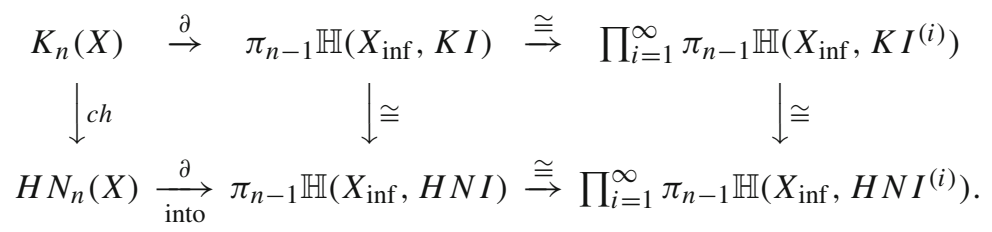

The two horizontal maps $\partial$ commute with $\psi^{k}$ because the operations $\psi^{k}$ are compatible with the fibration $K I(T) \rightarrow K(T) \rightarrow K(U)$ (and its $H N$ analogue) used in the proof of Theorem 5.3. Thus if $x \in K_{n}^{(i)}(X)$ then $\partial(x) \in \pi_{n-1} \mathbb{H}\left(X_{\mathrm{inf}}, K I^{(i)}\right)$, and a diagram chase shows that $\operatorname{ch}(x)$ is in $H N_{n}^{(i)}(X)$.

Corollary 7.2 For all $X$ in $\mathrm{Sch} / F$ and all $n$, the map ch $: K_{n}(X) \rightarrow H N_{n}(X)$ satisfies $\psi^{k}(c h)=k \cdot \operatorname{ch}\left(\psi^{k}\right)$, i.e., ch sends $K_{n}^{(i)}(X)$ to $H N_{n}^{(i)}(X)$.

Proof We proceed by downward induction on $n$, Theorem 7.1 being the base case $n \geq 1$. Given $x \in K_{n}(X)$, the element $\{x, t\}$ of $K_{n+1}\left(X \times \mathbb{G}_{m}\right)$ satisfies $\psi^{k} \operatorname{ch}(\{x, t\})=$ $k \cdot \operatorname{ch}\left(\psi^{k}\{x, t\}\right)$ by induction. But $\psi^{k}$ is multiplicative on $K$-theory, and the Chern character is multiplicative by [29, Sect. 5] [21], so by Example 0.2 we have:

$$
\operatorname{ch}\left(\psi^{k}\{x, t\}\right)=\operatorname{ch}\left(\psi^{k} x\right) \cdot \operatorname{ch}\left(\psi^{k} t\right)=k \operatorname{ch}\left(\psi^{k} x\right) \cdot \operatorname{ch}(t) .
$$

Although the Adams operations $\psi^{k}$ are not multiplicative on $H N$, the operations $\bar{\lambda}^{k}=(-1)^{k-1} \lambda^{k}$ are multiplicative because they commute with the shuffle product (see [40, 4.5.14, 5.1.14]). Since the Adams operations satisfy $\psi^{k}=k \bar{\lambda}^{k}$, we have $k \psi^{k}(a \cdot b)=\psi^{k} a \cdot \psi^{k} b$ in $H N$. Hence

$\left.k \psi^{k}(\operatorname{ch}\{x, t\})=k \psi^{k}(\operatorname{ch}(x) \cdot \operatorname{ch}(t))\right)=\psi^{k} \operatorname{ch}(x) \cdot \psi^{k} \operatorname{ch}(t)=k^{2} \psi^{k} \operatorname{ch}(x) \cdot \operatorname{ch}(t)$.

The result follows, because we can divide by $k$ in $H N_{*}$, and multiplication by $\operatorname{ch}(t)$ is an injection from $H N_{n}(X)$ into $H N_{n+1}\left(X \times \mathbb{G}_{m}\right)$.

Proof of Theorem 0.1 Every element of $K_{n}(A)$ comes from $K_{n}\left(A_{0}\right)$ for some subalgebra $A_{0}$ of finite type, so we may assume that $A$ is of finite type over a field $F$. If $n \leq 0$, the theorem follows from [53], and the case $n \geq 1$ is handled by Theorem 7.1 above (with $X=\operatorname{Spec} A$ ). 
Acknowledgments The authors would like to thank Rick Jardine for his help with fibrant replacements, especially in the proof of Theorem 4.9. We would also like to thank the referee for pointing out that the relative Chern character and the rational homotopy characters needed to be identified, a problem solved in [14].

Open Access This article is distributed under the terms of the Creative Commons Attribution Noncommercial License which permits any noncommercial use, distribution, and reproduction in any medium, provided the original author(s) and source are credited.

\section{A Appendix: Cathelineau's Theorem}

In this appendix, we correct the proof of Cathelineau's theorem [6]. Recall that Goodwillie's theorem [26] identifies the relative $K$-theory $K_{n}(A, I)$ and cyclic homology $H C_{n-1}(A, I)$ of a nilpotent ideal $I$ in a $\mathbb{Q}$-algebra $A$. Also recall that these groups are the direct sum of their $k^{i}$-eigenspaces $K_{n}^{(i)}(A, I)$ and $H C_{n}^{(i)}(A, I)$ for the Adams operation $\psi^{k}$.

Actually, Goodwillie gave two such isomorphisms, the relative Chern character $c h_{*}$ and the rational homotopy character $\rho_{*}$. These group isomorphisms are shown to be identical in [14], since the maps which induce them are naturally homotopic.

Cathelineau's Theorem A.1 Let I be a nilpotent ideal in a commutative $\mathbb{Q}$-algebra A. Then Goodwillie's isomorphism

$$
K_{n}(A, I) \cong H C_{n-1}(A, I)
$$

is an isomorphism of trivial $\gamma$-rings. That is, it identifies $K_{n}^{(i)}(A, I)$ and $H C_{n-1}^{(i-1)}$ (A,I).

Using the Science Citation Index to follow Ariadne's Thread, we see that Cathelineau's Theorem A.1 has been used in [15,16,19,21,34,51] and the present paper. In addition [40] and several other papers have cited it without using it: $[7,8,22$, $23,37,38,41,53]$.

The problem with the proof in [6] is that it references the unpublished Ogle-Weibel preprint [42], which mistakenly asserts in [42, 1.6] that the $G L(\mathbb{Q})$-invariant Chevalley-Eilenberg chain complex $x^{G L(\mathbb{Q})}(A, I)$ is quasi-isomorphic to a certain homotopy colimit. To fix it, we shall systematically use the combinatorial version $X(A, I)$ corresponding to the symmetric group $\Sigma_{\infty}$.

Step 1. Most of this step is presented in [40]. If $A$ is a ring with unit, and $T_{n}(A)$ is the subgroup of $G L_{n}(A)$ consisting of upper triangular matrices, then the Volodin space $X(A) \subset B G L(A)$ is the union of the spaces $X_{n}(A)=\bigcup_{\sigma \in \Sigma_{n}} B T_{n}^{\sigma}(A)$; Suslin proved in [46] that $X(A)^{+}$is contractible and that there is a homotopy fibration

$$
X(A) \rightarrow B G L(A) \rightarrow B G L(A)^{+} .
$$

If $I$ is an ideal in $A$, we may consider the subgroup $T_{n}(A, I)$ of $G L_{n}(A)$ consisting of matrices which are upper-triangular modulo $I$, i.e., the preimage of the upper triangular matrices $T_{n}(A / I)$. By definition, the space $X_{n}(A, I)$ is the union $\bigcup_{\sigma \in \Sigma_{n}} B T_{n}^{\sigma}(A, I)$. 
Let $\overline{G L}(A / I)$ denote the image of $G L(A)$ in $G L(A / I)$, and define $K(A, I)$ to be the homotopy fiber of $B G L(A)^{+} \rightarrow B \overline{G L}(A / I)^{+}$; by construction, $K(A, I)$ is a connected space whose homotopy groups $\pi_{n} K(A, I)$ are the relative $K$-groups $K_{n}(A, I)$ for all $n \geq 1$. The following theorem is Theorem 6.1 of [42]; it is cited in [6] as well as in [2]. As usual, $X(A, I)$ denotes the union of the $X_{n}(A, I)$.

Theorem A.3 If I is an ideal in A, there are homotopy fibrations

$$
\begin{aligned}
X(A, I) & \rightarrow B G L(A) \rightarrow B \overline{G L}(A / I)^{+}, \\
X(A) & \rightarrow X(A, I) \rightarrow K(A, I) .
\end{aligned}
$$

Moreover, there is a homology isomorphism $X(A, I) \rightarrow K(A, I)$ and a homotopy equivalence $X(A, I)^{+} \simeq K(A, I)$.

Proof The original proof of Theorem 6.1 in [42] works, and is reproduced on page 361 of [40].

Lemma A.4 If $\sigma \in \Sigma_{n+1}$, the canonical embedding $i_{n}: X_{n} \rightarrow X_{n+1}$ and the embedding $\sigma i_{n} \sigma^{-1}$ are naturally homotopic. Hence $\Sigma_{\infty}$ acts trivially on $H_{*} X(A, I)$.

Proof As noted in [42, 3.1] [25, I.5], Suslin's argument in [47, 1.5] goes through.

Corollary A.5 The direct sum of matrices makes $X(A, I)^{+}$into an $H$-space. In particular, $H_{*}(X(A, I) ; \mathbb{Q})$ is a Hopf algebra.

Proof Lemma A.4 is the ingredient needed for the classical proof to go through. (cf. [42, Remark 6.1.1] and the proof in [40, 11.3.4]).

In order to view $X(A, I)$ as a homotopy colimit, we need to expand the indexing set from $\Sigma_{n}$ to the collection $\mathcal{A}_{n}$ of all partial orders $\sigma$ of $\{1, \ldots, n\}$, so that the family of subgroups $T^{\sigma}$ is closed under intersection. This expands $\Sigma_{\infty}=\cup \Sigma_{n}$ to $\mathcal{A}=\cup \mathcal{A}_{n}$, and is the standard adjustment described in [25, p. 386], [42, 1.5] and [2, 1.2]. The point is that the natural map of simplicial chain complexes

$$
\underset{\mathcal{A}_{n}}{\operatorname{hocolim}} C_{*}\left(B T_{n}^{\sigma}(A, I)\right) \rightarrow C_{*}\left(X_{n}(A, I)\right)
$$

is a quasi-isomorphism for all $n$, including $n=\infty$. The proof of Claim III.9 in [25] goes through in this setting, as does the proof of [42, 1.4].

Step 2. On pp. 391-393 of [25], Goodwillie considered the Lie algebra $t_{n}(A)$ of strictly upper triangular matrices and introduced the sum $x_{n}(A)=\sum_{\sigma \in \Sigma_{n}} C_{*} \mathrm{t}_{n}^{\sigma}(A)$ of the Chevalley-Eilenberg chain complexes as a useful homological tool. He also introduced the subgebra $\mathfrak{t}_{n}^{\sigma}(A)$ for each partial order $\sigma$ of $\{1, \ldots, n\}$ and proved [25, Claim III.9] that the canonical map hocolim $\mathcal{A}_{n} C_{*} \mathfrak{t}_{n}^{\sigma}(A) \rightarrow x_{n}(A)$ is a quasi-isomorphism.

There are quasi-isomorphisms $C_{*}\left(T_{n}^{\sigma}(A), \mathbb{Q}\right) \stackrel{\simeq}{\longrightarrow} C_{*} \mathrm{t}_{n}^{\sigma}(A)$, arising from rational homotopy theory, which are natural in $n$ and $\sigma$. They were used by Goodwillie in [25, 
p. 392] and shown to be natural in [48, 5.11]. As observed on p. 85 of [48], naturality implies that if $\mathbb{Q} \subseteq A$ there is a natural quasi-isomorphism

$$
C_{*}\left(X_{n}(A), \mathbb{Q}\right) \stackrel{\simeq}{\longrightarrow} x_{n}(A)
$$

If $I$ is a nilpotent ideal in $A$, and $\sigma$ is a partial ordering of $\{1, \ldots, n\}$, we may consider the nilpotent Lie subalgebra $\mathfrak{t}_{n}^{\sigma}(A, I)$ of $\mathfrak{g l}_{n}(A)$ consisting of matrices whose reductions modulo $I$ are in $\mathfrak{t}_{n}^{\sigma}(A / I)$. The rational homotopy theoretic argument given by Goodwillie on pp. 392-3 of [25] (and made natural in [48, 5.11]) works for any nilpotent Lie algebra over $\mathbb{Q}$, and so works in this context to yield canonical quasiisomorphisms

$$
C_{*}\left(T_{n}^{\sigma}(A, I), \mathbb{Q}\right) \stackrel{\simeq}{\longrightarrow} C_{*} \mathfrak{t}_{n}^{\sigma}(A, I)
$$

Now consider the chain complex $x_{n}(A, I)=\sum_{\sigma} C_{*}\left(\mathfrak{t}_{n}^{\sigma}(A, I)\right)$. The following lemma repairs the mistake in $[42,1.6]$, which is also a gap in the sketch of $[40$, 11.3.15].

Lemma A.9 The map hocolim $\mathcal{A}_{n} C_{*} \mathrm{t}_{n}^{\sigma}(A, I) \rightarrow x_{n}(A, I)$ is a quasi-isomorphism.

Proof Choosing a basis for $I$ and completing it to a basis of $A$, we get a basis of $C_{*} \mathfrak{g l}_{n}(A)$ which restricts to a basis $B_{*}(\sigma)$ of each $C_{*} \mathfrak{t}_{n}^{\sigma}(A, I)$, and this family of chain complexes is closed under intersection. Thus for each $p$ the functor $\sigma \mapsto C_{p} t_{n}^{\sigma}(A, I)$ is the free vector space on the underlying basis functor $B_{p}$ from $\mathcal{A}_{n}$ to sets. For each basis element $b \in \cup B_{p}(\sigma)$ there is a unique minimal partial order $\alpha$ so that $b \in B_{p}(\alpha)$. This is enough for the proof of Claim III.9 in [25] to go through.

Remark $A .10$ The construction of the basis $B_{p}(\sigma)$ is also enough for the following proof to work; it is the original proof of $[42,1.4]$. For each $q$, the simplicial homotopy

$$
h_{j}\left(b, \sigma_{0} \rightarrow \cdots\right)=\left(b, \alpha=\cdots \alpha \rightarrow \sigma_{j} \rightarrow \cdots\right)
$$

from the identity of hocolim $C_{q}$ to the retraction onto the subgroup $x_{q}(A, I)$ shows that $H_{p}$ (hocolim $\left.C_{q}\right)$ is: $x_{q}(A, I)$ if $p=0$ and zero otherwise. Thus the spectral sequence $E_{p q}^{1}=H_{p}\left(\right.$ hocolim $\left.C_{q}\right) \Rightarrow H_{p+q}$ hocolim $C_{*}$ degenerates at $E^{2}$ to yield the conclusion $H_{p} x_{n}(A, I) \cong H_{p}$ hocolim $C_{*}$ of Lemma A.9.

Theorem A.11 There is a natural isomorphism

$$
H_{*}\left(X_{n}(A, I), \mathbb{Q}\right) \cong H_{*} x_{n}(A, I)
$$

for each $n$, induced by $\Sigma_{n}$-equivariant quasi-isomorphisms:

$$
\begin{aligned}
C_{*}\left(X_{n}(A, I), \mathbb{Q}\right) & \simeq \underset{\mathcal{A}_{n}}{\operatorname{hocolim}} C_{*}\left(T_{n}^{\sigma}(A, I), \mathbb{Q}\right) \\
& \simeq \underset{\mathcal{A}_{n}}{\operatorname{hocolim}} C_{*} \mathfrak{t}_{n}^{\sigma}(A, I) \simeq x_{n}(A, I) .
\end{aligned}
$$


Proof Combine (A.6) and the homotopy colimit of (A.8) with Lemma A.9.

Remark A.12 The natural maps in (A.8) assemble directly to yield the natural quasiisomorphism $\phi: C_{*}\left(X_{n}(A, I), \mathbb{Q}\right) \stackrel{\simeq}{\longrightarrow} x_{n}(A, I)$. This follows from repeated use of Mayer-Vietoris sequences, as observed in [48, p. 85]. A second hocolim-free proof of Theorem A.11 is sketched in [40, 11.3.15].

The composition $\rho$ of the Hurewicz map $K(A, I) \stackrel{h}{\longrightarrow} C_{*} K(A, I)$, the homotopy equivalence of Theorem A.3, the map $\phi$ of Remark A.12 and the Loday-Quillen map $\theta: x(A, I) \rightarrow H C(A, I)[1]$ (defined in $[40,10.2 .3 / 11.3 .12])$ and $B: H C(A, I)[1] \simeq$ $H N(A, I)$ is:

$$
K(A, I) \stackrel{h}{\rightarrow} C_{*} K(A, I) \stackrel{\simeq}{\leftarrow} C_{*} X(A, I) \stackrel{\phi}{\rightarrow} x(A, I) \stackrel{\theta}{\rightarrow} H C(A, I)[1] \stackrel{B}{\rightarrow} H N(A, I) .
$$

Here the cyclic homology complex is taken over $k=\mathbb{Q}$, and the non-negative chain complexes are regarded as simplicial sets using the Dold-Kan correspondence. We write $c h_{\text {rht }}^{-}$for the composition $B \theta \circ \phi: C_{*} X(A, I) \rightarrow H N(A, I)$ in (A.13); it is natural in $A$ and $I$.

Definition A.14 ([26]; cf. [40, 11.3.1]) Let $I$ be a nilpotent ideal in a $\mathbb{Q}$-algebra $A$. The map $\rho: K(A, I) \rightarrow H C(A, I)[1]$ of (A.13) is called the rational homotopy theory character. Goodwillie proved in [26] that $c h_{\text {rht }}^{-}$and hence $\rho$ are homotopy equivalences for all $(A, I)$. It is the map invoked by Cathelineau in [6, p. 600].

Here is the correction to Proposition 4.3 of [42], which is also the conclusion (1.2.4) of Aboughazi-Ogle [2]. Since their proof formally relies on some assertions in [42], this theorem also fills in the details of the presentation in [2].

Theorem A.15 $[40,11.3]$ If I is a nilpotent ideal in a $\mathbb{Q}$-algebra $A$ then the map $\rho$ induces isomorphisms for all $m \geq 1$ :

$$
K_{m}(A, I) \cong \operatorname{Prim} H_{m}\left(C_{*} X(A, I), \mathbb{Q}\right) \cong \operatorname{Prim} H_{m} x(A, I) \cong H C_{m-1}(A, I) .
$$

Proof We need only verify that the proof of (1.2.4) in [2] works mutatis mutandis. Equation (1.2.1) in [2] is the combination of (A.6) and Theorem A.3. Equation (1.2.2) in [2] is just Theorem A.11. Given these substitions and Lemma A.4, the proof of Claim 1.2.3 in [2] is valid. Therefore Theorem 1.1.12 of [2] applies to the complex $x(A, I)$, as asserted in [2], to yield the desired isomorphisms.

We will need the following result, proven in [14, 6.5]. The relative Chern character ch $: K(A, I) \rightarrow H N(A, I)$ was defined by Goodwillie in [26, II.3.3] as the composition of the map

$$
K(A, I) \stackrel{h}{\longrightarrow} C_{*} K(A, I) \stackrel{\sim}{\leftarrow} C_{*} X(A, I)
$$

of (A.13) and a natural map $c h^{-}: C_{*} X(A, I) \rightarrow H N(A, I)$ (see $[40,11.4 .6]$ for the definition of $\left.c h^{-}\right)$. 
Proposition A.16 $[14,6.5 .1]$ Let I be a nilpotent ideal in a $\mathbb{Q}$-algebra A. Then the map $\mathrm{ch}^{-}$is naturally chain homotopy equivalent to $\mathrm{ch}_{\mathrm{rht}}^{-}$. Hence the relative Chern character ch and the rational homotopy character $\rho$ (composed with $H C(A, I)[1] \simeq$ $H N(A, I))$ are homotopic for each $(A, I)$.

Step 3. Cathelineau's paper [6] refers to the preprint [42] in the following places.

1. On p. 597, Cathelineau cites [42, 2.3] for the isomorphism $H_{*} \mathfrak{t}_{n}(A, I) \cong H_{*}$ $\left(T_{n}(A, I), \mathbb{Q}\right)$. This isomorphism is rederived in (A.8) above, and in [40, 11.3.14].

2. Set $G=G L(\mathbb{Q})$. On p. 599, the space $X^{G}(A, I)=\bigcup_{g \in G} B T(A, I)^{g}$ and the chain complex $x^{G}(A, I)=\sum_{g \in G} C_{*}\left(\mathfrak{t}^{g}\right)$ of [42] are introduced, and then [42, 2.4] is cited for the existence of a $G$-equivariant isomorphism $\Phi^{G}: H_{*}\left(x^{G}(A, I)\right) \rightarrow$ $H_{*}\left(X^{G}(A, I)\right)$ in the proof of Lemma 2 .

The existence of $\Phi^{G}$ is unclear. As explained above, we need to replace $X^{G}(A, I)$ by the space $X(A, I)$, and $x^{G}(A, I)$ by the chain complex $x(A, I)$. By appealing to Theorem A.11 above, we get the required isomorphism $H_{*}(x(A, I) \cong$ $H_{*}(X(A, I))$. This corrects the proof of Lemma 2 of [6].

3. In the definition of $\lambda^{k}$ ([6], bottom of p. 599), Cathelineau cites [42, 3.2] for the fact that $G$ acts trivially on the homology of $X^{G}(A, I)$ and $x^{G}(A, I)$, so that the maps $\lambda_{n}^{k}$ are compatible with inductive limits. This is not a problem any more, by Lemma A.4 and Theorem A.11.

4. He cites $[42,6.1]$ for the fact that $X^{G}(A, I) \rightarrow K(A, I)$ is a homology isomorphism. This is replaced by Theorem A.3 above.

5. In the middle of p. 600 , he cites $[42,4.3]$ that the primitives of $H_{*}(X)$ agree with $H C_{*-1}(A, I)$. This is replaced by Theorem A.15.

6. The $H$-space structure on $X(A, I)^{+}$, used to define the topological maps $\lambda_{n}^{k}$ from $X_{n}(A, I)$ to $X(A, I)^{+}$on p. 601, is deduced from Remark 6.1.1 of [42]. This is addressed in Corollary A.5 above.

7. In order to see that $\lambda_{n+1}^{k}$ is freely homotopic to $i_{n} \circ \lambda_{n}^{k}$, Cathelineau invokes the argument of [42, 3.1]. In fact, these maps differ by conjugation by an element of $\Sigma_{n+1}$, so this follows from Lemma A.4 above.

8. Cathelineau's Remark 2.5 points out that, while compatibility with the $\lambda$-structures is proven for the isomorphism $K_{n}(A, I)_{\mathbb{Q}} \cong H C_{n-1}(A, I)$ of $[42,6.2]$, which is the rational homotopy character (A.14), it agrees with the isomorphism $\rho$ constructed by Goodwillie in [26].

\section{B Appendix: Space-level versions of Cathelineau's Theorem}

Cathelineau's Theorem A.1 concerns the $K$-groups $K_{m}(A, I)=\pi_{m} K(A, I)$ of a nilpotent ideal in a ring. In this Appendix, we develop a space-level version (Theorem B.8) of Cathelineau's Theorem. That is, for every nilpotent sheaf of ideals $I$, the rational homotopy character (A.13) from the simplicial presheaf $K(\mathcal{O}, I): U \mapsto$ $K(\mathcal{O}(U), I(U))$ to $H C(\mathcal{O}, I)[1] \cong H N(\mathcal{O}, I)$ preserves the $\lambda$-filtration. By Proposition A.16, the same is true for the relative Chern character up to homotopy. Theorem B.11, the spectrum analogue of Theorem B.8, is needed for the proof of Theorem 6.3, which in turn is a key ingredient in the proof of Theorem 7.1; our Main Theorem 0.1 
is a special case of Theorem 7.1. Theorem B.8 is also used for the scheme-theoretic Theorem 6.1.

We will work with a presheaf model (B.1) of the affine rational homotopy character (A.13) of a sheaf $I$ of nilpotent ideals. The remaining steps, which were alluded to in Step 3 of Appendix A, follow Cathelineau's construction in [6]. Compatible presheaf operations $\Lambda^{k}$ and $\lambda^{k}$ are constructed on the $X_{n}$ and $x_{n}$ in (B.3) and B.5. The full compatibility is given in Theorem B.8.

We need to re-introduce some of the notation used in Appendix A. For each $n$, any ideal $I$ determines a sheaf of subgroups $T_{n}(\mathcal{O}, I)$ of $G L_{n}(\mathcal{O})$; we form the simplicial subsheaf $X_{n}(\mathcal{O}, I)=\bigcup_{\sigma \in \Sigma_{n}} B T_{n}^{\sigma}(\mathcal{O}, I)$ of $B G L_{n}(\mathcal{O})$ by conjugating by permutation matrices. By abuse of notation, we shall write $B G L(\mathcal{O})^{+}$for the functorial fibrant model $\mathbb{Z}_{\infty} B G L(\mathcal{O})$ of the plus construction, and write $K(\mathcal{O}, I)$ for the homotopy fiber of $B G L(\mathcal{O})^{+} \rightarrow B G L(\mathcal{O} / I)^{+}$. Recall from Theorem A.3 that there is a homology isomorphism from $X(\mathcal{O}, I)=\bigcup X_{n}(\mathcal{O}, I)$ to $K(\mathcal{O}, I)$, i.e., the natural map $C_{*} X(\mathcal{O}, I) \rightarrow C_{*} K(\mathcal{O}, I)$ is a quasi-isomorphism when evaluated at any $U$. Therefore $K_{*}(\mathcal{O}, I) \cong \operatorname{Prim} H_{*}(X(\mathcal{O}, I))$.

The sheafification of Theorem A.11 (see A.12) yields a $\Sigma_{n}$-equivariant quasi-isomorphism: $\phi: C_{*} X_{n}(\mathcal{O}, I) \stackrel{\simeq}{\longrightarrow} x_{n}(\mathcal{O}, I)$. This yields the presheaf version of the rational homotopy character (A.13):

$$
K(\mathcal{O}, I) \stackrel{h}{\longrightarrow} C_{*} K(\mathcal{O}, I) \stackrel{\simeq}{\leftarrow} C_{*} X(\mathcal{O}, I) \stackrel{\simeq}{\longrightarrow} x(\mathcal{O}, I) \rightarrow H C(\mathcal{O}, I)[1] .
$$

By Proposition A.16, the map $C_{*} X(\mathcal{O}, I) \rightarrow H C(\mathcal{O}, I)[1] \simeq H N(\mathcal{O}, I)$ in (B.1) is naturally homotopy to $\mathrm{ch}^{-}$and hence (B.1) is homotopy equivalent to the presheaf form of the relative Chern character.

In order to invert the backwards arrow in (B.1), we use the global projective closed model structure on simplicial presheaves of sets (discussed in Appendix C); this is the simplicial closed model structure in which a presheaf map $f$ is a fibration or a weak equivalence if $f(U)$ is one for each Zariski open $U$. Let $K(\mathcal{O}, I)^{\prime}$ be the cofibrant replacement for $K(\mathcal{O}, I)$, and factor the backwards map in (B.1) as $C_{*} K(\mathcal{O}, I) \stackrel{\simeq}{\longleftarrow} C \stackrel{\simeq}{\longleftarrow} C_{*} X(\mathcal{O}, I)$. Then $h$ lifts to a map $h^{\prime}: K(\mathcal{O}, I)^{\prime} \rightarrow C$ and, since $x(\mathcal{O}, I)$ is fibrant, (B.1) lifts to a map

$$
K(\mathcal{O}, I)^{\prime} \stackrel{h^{\prime}}{\longrightarrow} C \stackrel{\simeq}{\longrightarrow} x(\mathcal{O}, I) \rightarrow H C(\mathcal{O}, I)[1]
$$

Since the relative Chern character $c h$ is defined using the same backwards arrow, the remarks after (B.1) show that $c h$ lifts to a map $K(\mathcal{O}, I)^{\prime} \stackrel{h^{\prime}}{\longrightarrow} C \rightarrow H N(\mathcal{O}, I)$, homotopy equivalent to (B.2), followed by $H C(\mathcal{O}, I)[1] \simeq H N(\mathcal{O}, I)$.

Our next step is to construct the operations $\lambda^{k}$ on $C_{*} X_{n}(\mathcal{O}, I)$ and $x_{n}(\mathcal{O}, I)$. Following Cathelineau, the exterior power operations determine group maps $\Lambda_{\times, n}^{k}$ from $T_{n}(\mathcal{O}, I)$ to $T_{\left(\begin{array}{c}n \\ k\end{array}\right)}(\mathcal{O}, I)$ and these induce maps of simplicial sheaves

$$
\Lambda_{\times, n}^{k}: X_{n}(\mathcal{O}, I) \rightarrow X_{\left(\begin{array}{c}
n \\
k
\end{array}\right)}(\mathcal{O}, I)
$$


The exterior power operations also determine Lie algebra maps $\Lambda_{+, n}^{k}$ from $\mathfrak{t}_{n}(\mathcal{O}, I)$ to $\mathfrak{t}_{\left(\begin{array}{c}n \\ k\end{array}\right)}(\mathcal{O}, I)$ and hence chain maps on $x_{n}(\mathcal{O}, I)$. These two constructions are compatible in the following sense. Let $h: X \rightarrow C_{*} X$ denote the Hurewicz map; as in (A.13), we regard non-negative chain complexes as spaces by Dold-Kan.

Lemma B.4 Let I be a nilpotent sheaf of ideals on $T$, and $n, k$ natural numbers. The following diagram commutes:

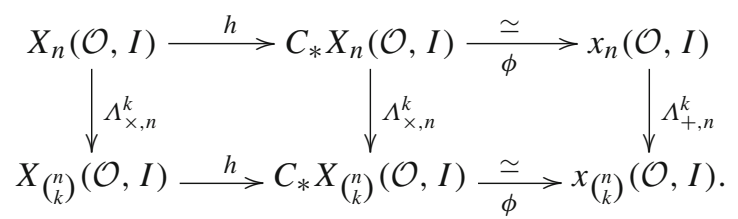

Proof This is immediate from the naturality of the map (A.8); see [48, 5.11]. The left square commutes by naturality of the Hurewicz map $h$ (see [54, 8.3.9]).

Now the homology of $C_{*} X(\mathcal{O}, I)$ is a Hopf algebra by Corollary A.5, and the same is true for $x(\mathcal{O}, I)$ by (A.7). Composing $\Lambda_{n}^{k}$ with the (chain-level) antipode, we can define maps $-\Lambda_{n}^{k}$ on $C_{*} X_{n}(\mathcal{O}, I)$ and $x_{n}(\mathcal{O}, I)$. Combining with the direct sum of matrices, we can define maps $\lambda_{n}^{k}=\oplus \pm \Lambda_{n}^{i}$ on $C_{*} X_{n}(\mathcal{O}, I)$ and $x_{n}(\mathcal{O}, I)$, taking values in the stable complexes $C_{*} X(\mathcal{O}, I)$ and $x(\mathcal{O}, I)$. (The number of times the factor $\pm \Lambda_{n}^{i}$ occurs in this expression is suppressed for legibility).

In the case of $X(\mathcal{O}, I)$ we can do better because $X(\mathcal{O}, I)^{+} \stackrel{\sim}{\longrightarrow} K(\mathcal{O}, I)$ is a weak equivalence by Theorem A.3. For this we let $X_{n}^{\prime}(\mathcal{O}, I)$ be a cofibrant replacement for $X_{n}(\mathcal{O}, I)$, so that the maps $\Lambda_{\times, n}^{k}$ of (B.3) lift to maps $X_{n}^{\prime}(\mathcal{O}, I) \rightarrow K(\mathcal{O}, I)^{\prime}$. Using the homotopy inverse on $K(\mathcal{O}, I)^{\prime}$ from Proposition C.2, we also have presheaf maps $-\Lambda_{\times, n}^{k}$. Using the $H$-space structure on $K(\mathcal{O}, I)^{\prime}$, we can combine the maps $\pm \Lambda_{\times, n}^{k}$ into a presheaf map $\lambda_{\times, n}^{k}: X_{n}^{\prime}(\mathcal{O}, I) \rightarrow K(\mathcal{O}, I)^{\prime}$ for each $n$. Given this stabilization, Lemma B.4 and (B.1) imply:

Proposition B.5 The following diagram commutes for each $n$ and $k$ :

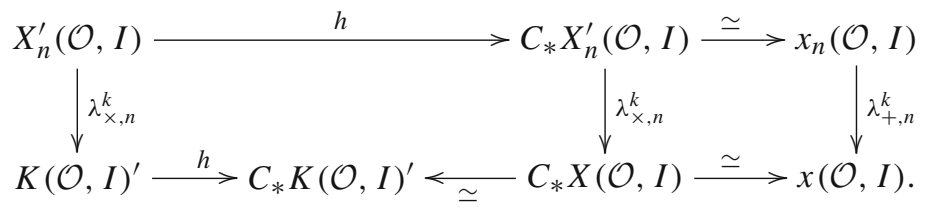

This completes the construction of the $\lambda^{k}$ on $X_{n}^{\prime}(\mathcal{O}, I)$ and $x_{n}(\mathcal{O}, I)$.

We may of course pass to the inductive limit over $n$ in the right square of (B.5), defining compatible operations on $C_{*} X^{\prime}(\mathcal{O}, I)$ and $x(\mathcal{O}, I)$, a passage made possible because the symmetric group action is homologically trivial by Lemma A.4. (This is observed in [6, Lemma 3] and in Step 3(3) of Appendix A to this paper).

We may also perform this stabilization on $X_{n}^{\prime}(\mathcal{O}, I)$. Indeed, each map $\lambda_{n}^{k}$ agrees with the composition $X_{n}^{\prime}(\mathcal{O}, I) \rightarrow X_{n+1}^{\prime}(\mathcal{O}, I) \rightarrow K(\mathcal{O}, I)$ up to conjugation by 
an element of $\Sigma_{\infty}$. As argued by Cathelineau on p. 601 of [6], using $S t(\mathbb{Z})$ in place of $\operatorname{St}(A)$, there are natural based homotopies between them, so that they are even base-point homotopic as presheaf maps. Thus they define a map

$$
X(\mathcal{O}, I) \stackrel{\sim}{\longleftarrow} \operatorname{hocolim} X_{n}^{\prime}(\mathcal{O}, I) \stackrel{\lambda_{n}^{k}}{\longrightarrow} K(\mathcal{O}, I)
$$

The operations $\lambda_{+, n}^{k}$ in B.5 are compatible with the operations on cyclic homology. As observed by Cathelineau on p. 600 of [6], the maps $x_{n}(\mathcal{O}, I) \rightarrow \wedge^{*} g l_{n}(\mathcal{O}, I)$ and the Loday-Quillen map both commute with the operations $\lambda_{n}^{k}$, so we get commutative diagrams:

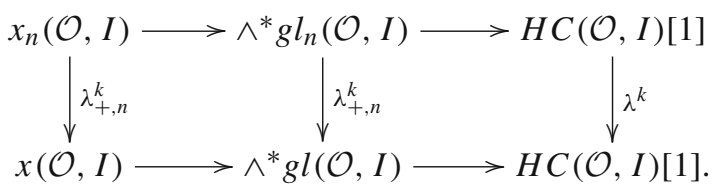

The triviality of the symmetric group action in Lemma A.4 extends to the presheaf level by naturality. This implies that the maps in B.5 and (B.7) are compatible (up to chain homotopy) with passage from $n$ to $n+1$. (This is observed on p.599 of [6] and in Step 3(3) of our Appendix A).

We are now ready to present a space-level version of Cathelineau's Theorem A.1:

Theorem B.8 The rational homotopy character (B.1) is compatible with the operations $\lambda^{k}$ defined on $K(\mathcal{O}, I)$ and $H C(\mathcal{O}, I)$, in the sense that there is a commutative diagram:

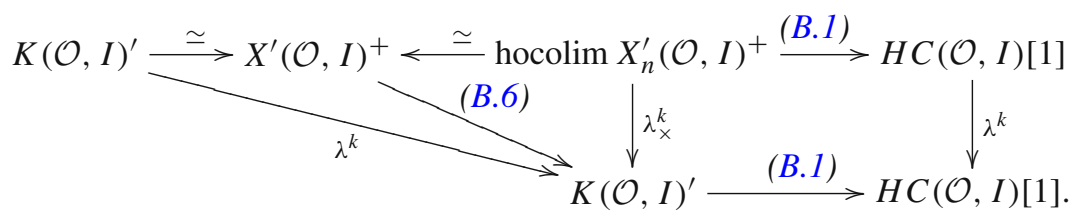

The relative Chern character ch $: K(\mathcal{O}, I) \longrightarrow H N(\mathcal{O}, I)$ is compatible with the $\lambda$-operations, up to the natural homotopy of Proposition A.16.

Proof The diagram (B.8) commutes because it is obtained by glueing (B.6) together with the homotopy colimits (over $n$ ) of the commutative diagrams (B.5) and (B.7), The upper left map is an inverse to the cofibrant replacement of the weak equivalence $X(\mathcal{O}, I)^{+} \rightarrow K(\mathcal{O}, I)$, constructed using Lemma C.1.

All that remains is to show that the operations $\lambda^{k}$ on $K(\mathcal{O}, I)$ displayed diagonally in (B.8) agree with the usual $\lambda$-operations induced from the operations on $B G L(\mathcal{O})^{+}$. To do this, we need to recall how the usual operations are defined.

As observed in $[6,2.1]$, the construction of the usual operations $\lambda^{k}$ may be chosen to follow the pattern described above. Briefly, one defines natural group maps $\Lambda_{n}^{k}$ on $G L_{n}(\mathcal{O})$ as in (B.3) and applies C.2 to the $H$-space structure on $B G L(\mathcal{O})^{+}$to 
obtain an $H$-inverse $\iota$ on a cofibrant replacement of $B G L(\mathcal{O})^{+}$, and then construct maps $\lambda_{n}^{k}$ on $B G L_{n}^{\prime}(\mathcal{O})$, as described before B.5. As in (B.6), this induces a map $\lambda^{k}$ on $B G L^{\prime}(\mathcal{O})$ and $B G L(\mathcal{O})^{+}$; finally this induces the relative operations $\lambda^{k}$ on $K(\mathcal{O}, I)$. (Compare with [45, p. 511]). By construction, the inclusions $X_{n}^{\prime}(\mathcal{O}, I) \subset B G L_{n}^{\prime}(\mathcal{O})$ are compatible with the operations $\lambda_{n}^{k}$ just defined, and the $\lambda^{k}$ defined in (B.6). Thus we have a commutative diagram:

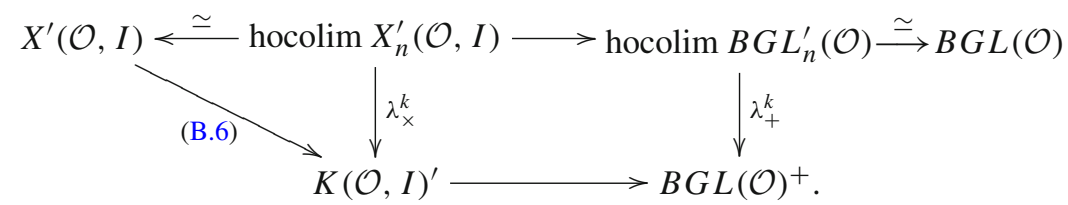

Applying the functorial +-construction $\mathbb{Z}_{\infty}$ converts $X^{\prime}(\mathcal{O}, I)$ and $B G L(\mathcal{O})$ into $K(\mathcal{O}, I)$ and $B G L(\mathcal{O})^{+}$in (B.8a), up to weak equivalence. As argued by Cathelineau on p. 602 of [6], the horizontal composites $K(\mathcal{O}, I) \rightarrow B G L(\mathcal{O})^{+}$are part of a presheaf of fibration sequences, with third term $B G L(\mathcal{O} / I)^{+}$. It follows that the map (B.6) does indeed induce the usual operation $\lambda^{k}$ on $K(\mathcal{O}, I)$, as claimed.

Remark B.9 Since the relative Chern character is multiplicative, it follows from Theorem B.8 that there is a similar compatibility result (up to homotopy) for polynomials in the $\lambda^{i}$. For example, the Adams operations $\psi^{k}$ are defined as polynomials; by [36, 5.3], $\psi^{k}=(-1)^{k-1} k \lambda^{k}$ on $\pi_{*} K(\mathcal{O}, I)$ and $H C_{*}(\mathcal{O}, I)$. Hence the Adams operations are compatible with the relative Chern character up to natural homotopy.

In order to extend Theorem B.8 to presheaves of spectra, recall that we can regard chain complexes as spectra, and morphisms of chain complexes as morphisms of spectra (see [52, p. 552]). In this way, $H C(\mathcal{O}, I)[1]$ is a presheaf of (connective) spectra and the $\lambda^{k}$ are spectrum endomorphisms of $H C(\mathcal{O}, I)$ [1].

In a parallel abuse of notation, we shall write $K(\mathcal{O}, I)$ for the presheaf of spectra whose initial space is the space $K(\mathcal{O}, I)$. Note that the a priori non-connective $K$-theory spectrum obtained by evaluation at any $U$ just happens to be connective because it is the relative $K$-theory spectrum of a nilpotent ideal. It is easy to see that the rational homotopy character and the relative Chern character $c h: K(\mathcal{O}, I) \longrightarrow H N(\mathcal{O}, I)$ are both morphisms of spectra, and that $c h$ is a homotopy equivalence of spectra by Goodwillie's Theorem [26]. (See [50, Exercise 9.10]; the key is that $c h$ is multiplicative by [29, Sect. 5]). Using the main result of [14], it is easy to see that these morphisms are homotopic. Combining these facts with Theorem B.8 and Remark B.9, we see that we have proven:

Corollary B.10 When I is a nilpotent sheaf of ideals, $\lambda^{k}$ and $\psi^{k}$ are morphisms of presheaves of spectra, from $K(\mathcal{O}, I)^{\prime}$ to $K(\mathcal{O}, I)$, and commute with ch up to natural homotopy equivalence.

We conclude with an interpretation in terms of the eigen-components with respect to these operations. We fix $k \geq 2$, and define $K^{(i)}(\mathcal{O}, I)$ to be the cofibrant homotopy 
fiber of $\lambda^{k}+(-1)^{k} k^{i-1}: K(\mathcal{O}, I)^{\prime} \rightarrow K(\mathcal{O}, I)$. We leave it as an exercise to see that (up to homotopy equivalence) this is independent of the choice of $k>1$, and that our choice here of $\lambda^{k}$ rather than $\psi^{k}$ is immaterial (use Remark B.9).

Recall too from [40, 4.6.7 and 4.5.16] that the chain complex for cyclic homology breaks up into the direct product of subcomplexes $H C^{(i)}$ on which $\lambda^{k}+(-1)^{k} k^{i}$ and $\psi^{k}-k^{i+1}$ are acyclic. Passing to the associated Eilenberg-Mac Lane spectra, this means that $H C^{(i)}(\mathcal{O}, I)$ is the homotopy fiber of $\lambda^{k}+(-1)^{k} k^{i}$ on $H C(\mathcal{O}, I)$. A similar description holds for $H N(\mathcal{O}, I)$; see [40, 5.1.20]. Thus we have proven:

Theorem B.11 The rational homotopy character (B.1) induces maps on each eigencomponent $K^{(i)}(\mathcal{O}, I)$, fitting into a commutative square:

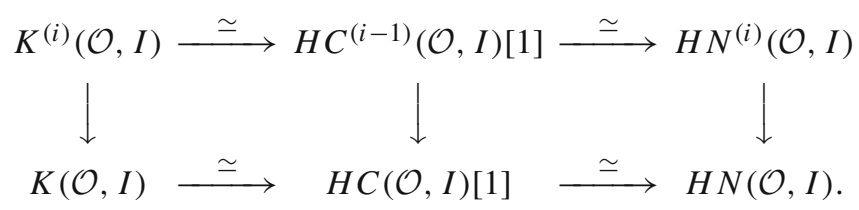

Using the fact that $H C(\mathcal{O}, I) \cong \prod_{i \geq 0} H C^{(i)}(\mathcal{O}, I)$, we see from Theorem B.11 that the maps $K^{(i)}(\mathcal{O}, I) \rightarrow K(\mathcal{O}, I)^{\prime}$ are split, and that we have a homotopy equivalence $K(\mathcal{O}, I)^{\prime} \stackrel{\simeq}{\longrightarrow} \prod_{i \geq 1} K^{(i)}(\mathcal{O}, I)$. Thus we deduce:

Corollary B.12 There is a homotopy commutative diagram:

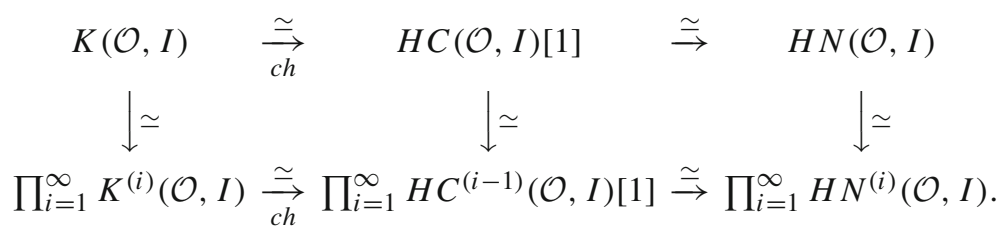

\section{Appendix: Simplicial presheaves of Sets}

In this Appendix we prove some elementary results about simplicial presheaves of sets, which are used in Appendix B. By a simplicial presheaf we mean a contravariant functor from a fixed small category $\mathbb{T}$ to the category of simplicial sets.

Lemma C.1 Let $f: E \rightarrow E^{\prime}$ be a weak equivalence in any model category, with $E$ fibrant and $E^{\prime}$ cofibrant. Then there is a map $g: E^{\prime} \rightarrow E$ such that $g f$ and $f g$ are weak equivalences.

Proof Consider the mapping cylinder $T$ of $f$, weak equivalent to $E^{\prime}$, constructed using the (CM5) factorization of $E \vee E^{\prime} \rightarrow E^{\prime}$ in the model structure. Since $E^{\prime}$ is cofibrant, $i: E \rightarrow T$ is a trivial cofibration. Since $E$ is fibrant, $i$ has a retract by axiom (CM4). But then the map $g: E^{\prime} \rightarrow T \rightarrow E$ has the desired properties; the homotopy from the identity on $E$ (which is $E \rightarrow T \rightarrow E$ ) to $g f$ is given by the map $T \stackrel{\sim}{\longrightarrow} E^{\prime} \stackrel{\sim}{\longrightarrow} T$; the homotopy from $f g$ to the identity on $E^{\prime}$ is similar. 
We say that a map $E \rightarrow E^{\prime}$ of simplicial presheaves is a (global) weak equivalence if each $E(U) \rightarrow E^{\prime}(U)$ is a homotopy equivalence of simplicial sets (or, more formally, a homotopy equivalence between the corresponding topological spaces). A global model structure on simplicial presheaves is one whose weak equivalences are the global weak equivalences.

Proposition C.2 Let $H$ be a fibrant presheaf of connected $H$-spaces, and $H^{\prime} \stackrel{\sim}{\rightarrow} H$ its cofibrant replacement in a global model structure. Then there is a presheaf map $\iota: H^{\prime} \rightarrow H^{\prime}$ such that each $H^{\prime}(U)$ is an $H$-group with homotopy inverse $\iota(U)$.

Proof Consider the shear map $\phi: H \times H \rightarrow H \times H$ defined by $\phi(x, y)=(x, x y)$. By [56, X.2.1], each $\phi(U)$ is a homotopy equivalence, i.e., $\phi$ is a global weak equivalence. If $C \stackrel{\sim}{\rightarrow} H \times H$ is a cofibrant replacement, then $C$ is fibrant (because $H \times H$ is) and the shear map lifts to a map $\phi: C \rightarrow C$. By C.1, there is a map $\psi: C \rightarrow C$ which is a homotopy inverse of $\phi$ on each $U$. Let $i_{1}: H^{\prime} \rightarrow C$ and $p r_{2}: C \rightarrow H^{\prime}$ be the cofibrant replacements of the inclusion $H=H \times * \rightarrow H \times H$ and the projection $H \times H \rightarrow * \times H=H$. By [56, III.4.17], each $H^{\prime}(U)$ is an $H$-group, via $H^{\prime}(U) \stackrel{\sim}{\longrightarrow} H(U)$; the composite $H^{\prime} \stackrel{i_{1}}{\longrightarrow} C \stackrel{\psi}{\longrightarrow} C \stackrel{p r_{2}}{\longrightarrow} H^{\prime}$ will be the desired map $\iota$ which is a homotopy inverse for each $H$-space $H^{\prime}(U)$.

We can do better using the global projective closed model structure, in which fibrations are defined objectwise; this simplicial closed model structure was introduced by Quillen in [43, Ch.II, Sect. 4, Th.4]. Indeed, the representable presheaves $h_{X}: U \mapsto \operatorname{Hom}_{\mathbb{T}}(U, X)$ form a family of small projective generators; see [4, pp. 314]. It follows that, for any $X$ in $\mathbb{T}$ and any cofibration of simplicial sets $K \longmapsto L$, the canonical arrow $h_{X} \otimes K \rightarrow h_{X} \otimes L$ is a cofibration,

The following result does not seem to be in the literature.

Theorem C.3 Assume that $\mathbb{T}$ has finite products. For the global projective closed model structure, the product of cofibrant presheaves is cofibrant.

Recall from the proof of [43, Ch.II, Sect. 4, Th.4] that every simplicial presheaf has a canonical cofibrant replacement $C^{(\infty)}$, in which the " $n$-skeleton" $C^{(n)}$ is obtained by attaching cells to $C^{(n-1)}$ of the form $h_{X} \otimes \Delta^{n}$ along maps $h_{X} \otimes \partial \Delta^{n} \rightarrow$ $C^{(n-1)}$.

Proof It suffices to assume that $C_{1}, C_{2}$ are canonical cofibrant presheaves and show that $C_{1} \times C_{2}$ is cofibrant. Since $h_{X} \times h_{Y}=h_{X \times Y}$, the result is true for representable presheaves, and in particular for the 0 -skeleton of $C_{1} \times C_{2}$. Inductively, suppose that $C^{n}=C_{1}^{(n)} \times C_{2}^{(n)}$ is cofibrant and that $C_{i}^{(n+1)}$ is obtained from $C_{i}^{(n)}$ by pushing out along coproducts of cells of the form $A_{i}=h_{X_{i}} \otimes \partial \Delta^{n} \longmapsto h_{X_{i}} \otimes \Delta^{n}=B_{i}$. Then

$$
C^{n} \rightarrow C^{(n+1)} \times C_{2}^{(n)} \rightarrow C^{(n+1)} \times C_{2}^{(n)} \cup_{C^{n}} C^{(n)} \times C_{2}^{(n+1)} \rightarrow C^{(n+1)} \times C_{2}^{(n+1)}
$$

is a cofibration since the first two maps are just pushouts along co-base extensions of $A_{i} \rightarrow B_{i}(i=1,2)$, and the final map is a co-base extension of $A_{1} \times A_{2} \longmapsto$ $B_{1} \times B_{2}$, which is a cofibration because it is a coproduct of maps $h_{X_{i} \times X_{j}} \otimes(K \longmapsto$ $L)$. 
Corollary C.4 If $H$ is a presheaf of connected fibrant $H$-spaces, its cofibrant replacement $H^{\prime}$ (in the global projective closed model structure) is a presheaf of $H$-groups.

Proof Since $H^{\prime} \times H^{\prime}$ is cofibrant by Theorem C.3, the product $H \times H \rightarrow H$ lifts to a product $H^{\prime} \times H^{\prime} \rightarrow H^{\prime}$, and the homotopy inverse $\iota: H^{\prime} \rightarrow H^{\prime}$ exists by Proposition C.2.

\section{References}

1. Artin, M., Mazur, B.: Étale Homotopy Theory. Springer Lecture Notes in Math., vol. 100 (1969)

2. Aboughazi, R., Ogle, C.: On Chevalley-Eilenberg and cyclic homologies. J. Algebra 166, 317-339 (1994)

3. Berthelot, P., Ogus, A.: Notes on Crystalline Cohomology. Princeton University Press, Princeton (1978)

4. Bousfield, A.K., Kan, D.: Homotopy Limits, Completions and Localizations. Lecture Notes in Math., vol. 304. Springer, Berlin (1987)

5. Bousfield, A.K., Friedlander, E.M.: Homotopy theory of $\Gamma$-spaces, spectra, and bisimplicial sets. Springer Lecture Notes in Math. 658, 80-130 (1978)

6. Cathelineau, J.-L.: $\lambda$-Structures in algebraic $K$-theory and cyclic homology. $K$-Theory 4, 591-606 (1991)

7. Cathelineau, J.-L.: Remarks on the differentials of the uniform polylogarithms. Ann. de l'Inst. Fourier 46, 1327-1347 (1996)

8. Cathelineau, J.-L.: Homology of tangent groups considered as discrete groups and scissors congruence. J. Pure Appl. Algebra 132, 9-25 (1998)

9. Cortiñas, G.: Infinitesimal $K$-theory. J. Reine Angew. Math. 503, 129-160 (1998)

10. Cortiñas, G.: The obstruction to excision in $K$-theory and cyclic homology. Invent. Math. 454, 143173 (2006)

11. Cortiñas, G.: De Rham and infinitesimal cohomology in Kapranov's model for noncommutative algebraic geometry. Compositio Mathematica 136, 171-208 (2003)

12. Cortiñas, G., Haesemeyer, C., Schlichting, M., Weibel, C.: Cyclic homology, $c d h$-cohomology and negative $K$-theory. Ann. Math. 167, 549-563 (2008)

13. Cortiñas, G., Haesemeyer, C., Weibel, C.: $K$-regularity, $c d h$-fibrant Hochschild homology and a conjecture of Vorst. J. AMS 21, 547-561 (2007)

14. Cortiñas, G., Weibel, C.: Relative Chern characters for nilpotent ideals. In: Proceedings of the Abel Symposium 2007. Available at http://www.math.uiuc.edu/K-theory/0901 (to appear)

15. Elbaz-Vincent, $\mathrm{Ph}$.: Homology of the special linear group and $K$-theory. Comptes Rendus Acad. Sci. (Paris) 322, 813-817 (1996)

16. Elbaz-Vincent, $\mathrm{Ph}$.: Homology of linear groups with coefficients in the adjoint action and $K$-theory. $K$-Theory 16, 35-50 (1999)

17. Feigin, B., Tsygan, B.: Additive $K$-theory. Springer Lecture Notes in Math. 1289, 67-209 (1987)

18. Geller, S., Reid, L., Weibel, C.: The cyclic homology and $K$-theory of curves. J. Reine Angew. Math. 393, 39-90 (1989)

19. Geller, S., Weibel, C.: Hodge decompositions of Loday symbols in $K$-theory and cyclic homology. $K$-Theory 8, 587-632 (1994)

20. Gillet, H., Soulé, C.: Filtrations on Higher $K$-theory. In: Algebraic $K$-theory. Proc. Symp. Pure Math., vol. 67, pp. 89-148. AMS, Providence (1999)

21. Ginot, G.: Formules explicites pour le caractère de Chern en $K$-théorie algébrique. Ann. Inst. Fourier (Grenoble) 54, 2327-2355 (2004)

22. Goncharov, A.: Volumes of hyperbolic manifolds and mixed Tate motives. J. Am. Math. Soc. 12, 569-618 (1999)

23. Goncharov, A.: Euclidean scissor congruence groups and mixed Tate motives over dual numbers. Math. Res. Lett. 11, 771-784 (2004)

24. Goodwillie, T.: Cyclic homology, derivations, and the free loopspace. Topology 24, 187-215 (1985)

25. Goodwillie, T.: On the general linear group and Hochschild homology. Ann. Math. 121, 383-407 (1985). Correction in Ann. Math. 124, 627-628 (1986) 
26. Goodwillie, T.: Relative algebraic $K$-theory and cyclic homology. Ann. Math. 124, 347-402 (1986)

27. Grothendieck, A.: Crystals and the de Rham cohomology of schemes. In: Grothendieck, A., Kuiper, N., (eds.) Dix exposés sur la cohomologie des schémas, pp. 306-358. Masson \& Cie, Paris (1968)

28. Hochschild, G., Kostant, B., Rosenberg, A.: Differential forms on regular affine algebras. Trans. AMS 102, 383-408 (1962)

29. Hood, C., Jones, J.: Some algebraic properties of cyclic homology groups. $K$-Theory 1, 361-384 (1987)

30. Jardine, J.F.: Simplicial presheaves. J. Pure Appl. Algebra 47, 35-87 (1987)

31. Jardine, J.F.: Stable homotopy theory of simplicial presheaves. Can. J. Math. 39, 733-747 (1987)

32. Jardine, J.F.: The Leray spectral sequence. J. Pure Appl. Algebra 61, 189-196 (1989)

33. Jardine, J.F.: Stacks and the homotopy theory of simplicial sheaves. HHA 3, 361-384 (2001)

34. Kantorovitz, M.R.: Adams operations and the Dennis trace map. J. Pure Appl. Alg. 144, $21-27$ (1999)

35. Kassel, C., Sletsjœ, A.B.: Base change, transitivity and Künneth formulas for the Quillen decomposition of Hochschild homology. Math. Scand. 70, 186-192 (1992)

36. Kratzer, C.: $\lambda$-structure en $K$-théorie algébrique. Comment. Math. Helv. 55, 233-254 (1980)

37. Krishna, A.: On $K_{2}$ of 1-dimensional rings. $K$-Theory 35, 139-158 (2005)

38. Krishna, A.: Zero-cycles on a threefold with isolated singularities. J. Reine Angew. Math. 594, 93-115 (2006)

39. Loday, J.-L.: Opérations sur l'homologie cyclique des algèbres commutatives. Invent. Math. 96, 205-230 (1989)

40. Loday, J.-L.: Cyclic homology. Grundlehren der Mathematischen Wissenschaften, vol. 301. Springer, Berlin (1992)

41. Loday, J.-L.: Algebraic $K$-theory and the conjectural Leibniz $K$-theory. $K$-Theory 30, 105-127 (2003)

42. Ogle, C., Weibel, C.: Cyclic homology and relative $K$-theory (1986, unpublished preprint)

43. Quillen, D.: Homotopical algebra. Lecture Notes in Math., vol. 43. Springer, Berlin (1967)

44. Quillen. D.: Cohomology of groups, pp. 47-51. Actes Cong. Int. Math. (1970)

45. Soulé, C.: Opérations en $K$-théorie algébrique. Can. J. Math. 37, 488-550 (1985)

46. Suslin, A.: On the equivalence of $K$-theories. Comm. Algebra 9, 1559-1566 (1981)

47. Suslin, A.: Stabilization in algebraic $K$-theory. Springer Lecture Notes in Math., vol. 966 (1983)

48. Suslin, A., Wodzicki, M.: Excision in algebraic $K$-theory. Ann. Math. 136, 51-122 (1992)

49. Thomason, R.W.: Algebraic $K$-theory and étale cohomology. Ann. Sc. Éc. Norm. Sup. (Paris) 18, 437-552 (1985)

50. Thomason, R.W., Trobaugh, T.: Higher algebraic $K$-theory of schemes and of derived categories. In: The Grothendieck Festschrift, vol. III. Progress in Math., vol. 88, pp. 247-436. Birkhäuser, Boston, Basél (1990)

51. Vigué-Poirrier, M.: Homology and $K$-theory of commutative algebras — characterization of completeintersections. J. Algebra 173, 679-695 (1995)

52. Weibel, C.A.: Nil $K$-theory maps to cyclic homology. Trans. AMS 303, 541-558 (1987)

53. Weibel, C.: Le caractère de Chern en homologie cyclique périodique. C.R. Acad. Sci. (Paris) 317, 867-871 (1993)

54. Weibel, C.A.: An Introduction to Homological Algebra. Cambridge University Press (1994)

55. Weibel, C.A.: Cyclic homology for schemes. Proc. AMS 124, 1655-1662 (1996)

56. Whitehead, G.W.: Elements of Homotopy Theory. Springer, Berlin (1978) 